2. To: (Receiving organization) B \& W Hanford Company

5. Proj./Prog-/Dept. /oiv.:

PFP/Z-361

8. Originator Remarks:
Transmittal of Tank $7-361$ Dose Rate Calculations Report

3. From: (originating organization)
FDNW

6. Design Authority/ Design Agent/Cog. Engr.: $N / A$ HhN 91100 t62 10/383/7000
11. Receiver Remarks: 11k. Design Basel ine Document?
[] Yes [X] no

\begin{tabular}{l|l} 
4. Related EDT No.: \\
$N / A$
\end{tabular}
$t \sqrt{ }$
9. Equip./Component No.:

N/A $N / A$

10. System/Bldo./Facility: 241-z-361 Tank

12. Major Assm. Dwg. Mo.: $N / A$

13. Pernit/Permit Applifation No.: N/A

14. Required Response Date: $N / A$

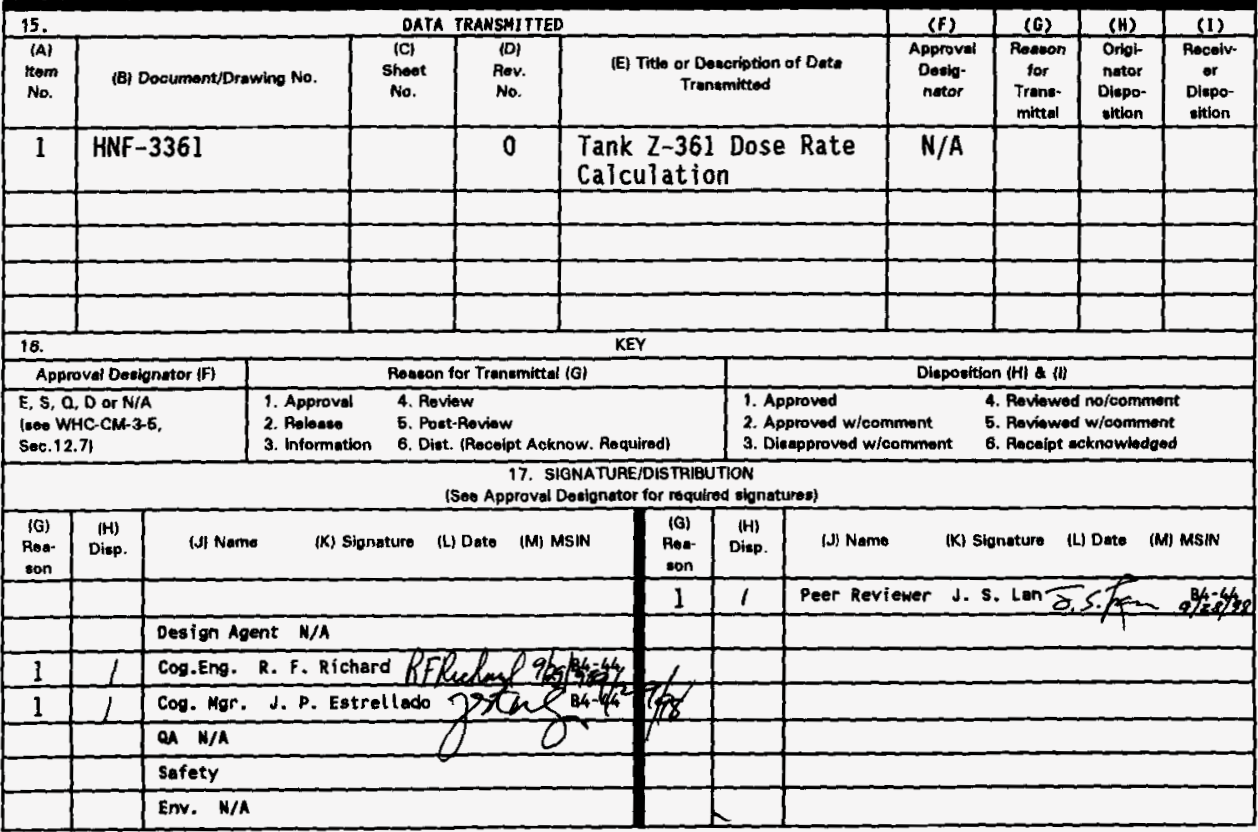

19.

Authorized Representative Deto for Feceiving Orgenization

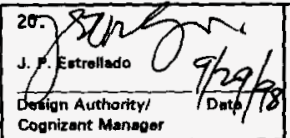

21. DOE APPROVAL (if required) Ctrl. No.

D) Aparoved

[] Approved w/comitents

[] Disapproved w/coniments

BD-7400-172-2 (05/96) GEF097 
HNF-3361, Rev. 0

\section{TANK Z-361 DOSE RATE CALCULATIONS}

Robert F. Richard

Fluor Daniel Northwest, Inc., Richland, WA 99352

U.S. Department of Energy Contract DE-AC06-96RL13200

EDT/ECN: 623014

UC: $510 \quad 10 / 383 / A 000$

Org Code: 04E00/403 Charge Code: K6216/HAN91100

B\&R Code: IK6A0D010A Total Pages: $60 \mathrm{kN}$ ro/5/se

Key Words: Dose rates, gamma ray, photon, neutron, Z-361 settling tank

Abstract: Gamma ray and neutron dose rate determination above the 6-inch riser and off the side of settling tank $Z-361$.

TRADEMARKS:

WordPerfect is a trademark of Corel Corporation.

Pentium is a trademark of Intel Corporation.

UNIX is a trademark licensed exclusively through XIOpen Company Ltd.

TRADEMARK DISCLAIMER. Reference herein to any specific commercial product, process, or service by trade name, trademark, manufacturer, or otherwise, does not necessarily constitute or imply its endorsement, recommendation, or favoring by the United States Government or any agency thereof or its contractors or subcontractors.

Printed in the United States of America. To obtain copies of this document, contact: Document Control Services, P.0. Box 950, Mailstop H6-08, Richland HA 99352, Phone (509) 372-2420;

fax (509) $376-4989$.
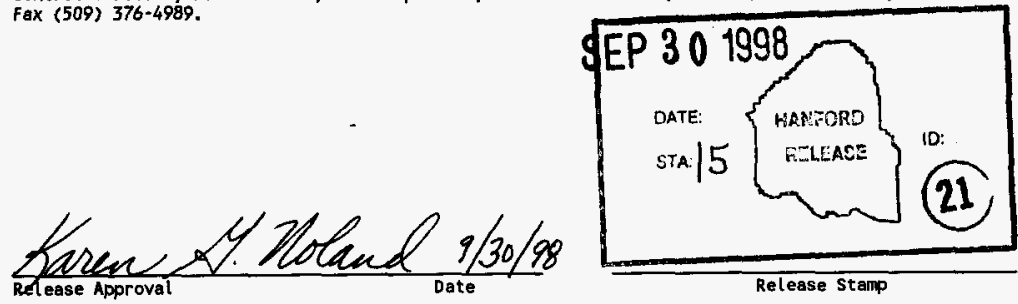

Approved for Public Release 
HNF-3361, Rev.0

\section{TANK Z-361 DOSE RATE CALCULATIONS}

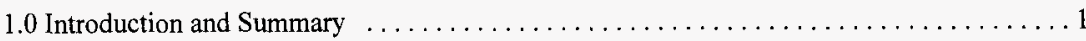

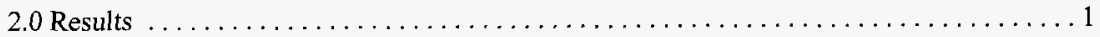

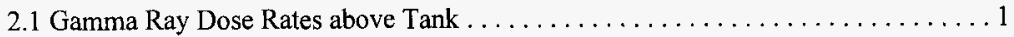

2.2 Gamma Ray Dose Rates at Side of Tank .........................

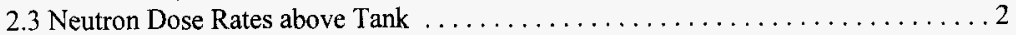

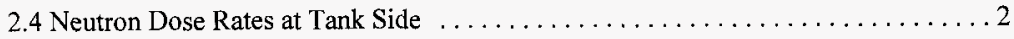

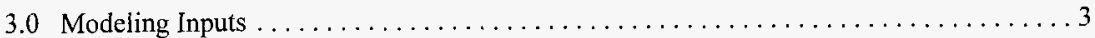

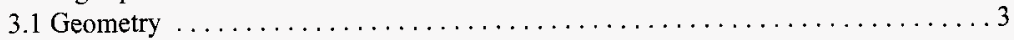

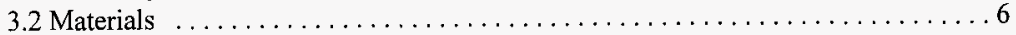

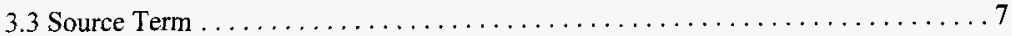

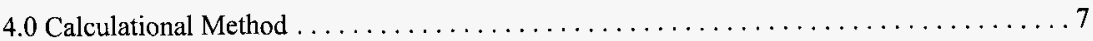

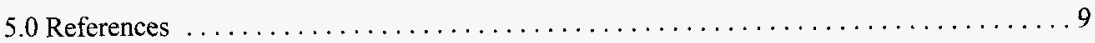

Appendix A: MCNP Input Files $\ldots \ldots \ldots \ldots \ldots \ldots \ldots \ldots \ldots \ldots \ldots \ldots \ldots \ldots \ldots \ldots \ldots$

Appendix B: Correspondence with Lee Carter $\ldots \ldots \ldots \ldots \ldots \ldots \ldots \ldots \ldots \ldots \ldots \ldots \ldots \ldots \ldots$

Appendix C: Checklist for Independent Technical Review ................. C-1 
HNF-3361, Rev.0

\section{TANK Z-361 DOSE RATE CALCULATIONS}

\subsection{INTRODUCTION AND SUMMARY}

Neutron and gamma ray dose rates were calculated above and around the 6-inch riser of tank Z-361 located at the Plutonium Finishing Plant. Dose rates were also determined off of one side of the tank. The largest dose rate $0.029 \mathrm{mrem} / \mathrm{h}$ was a gamma ray dose and occurred 76.2 $\mathrm{cm}$ (30 in.) directly above the open riser. All other dose rates were negligible.

\subsection{RESULTS}

The ANSI/ANS 1991 flux to dose conversion factor for neutrons and photons were used in this analysis (Reference 6). Dose rates are reported in units of $\mathrm{mrem} / \mathrm{h}$ with the calculated uncertainty shown within the parentheses.

\subsection{Gamma Ray Dose Rates above Tank}

At $76.20 \mathrm{~cm}$ ( $30 \mathrm{in}$.) directly above the top of the uncovered 6 inch riser, over an area extending radially to the riser radius, the gamma ray dose rate is $0.029 \mathrm{mrem} / \mathrm{h}$. At the same elevation but extending radially beyond the riser radius, the dose rate drops off by over a factor of 100 . A $2.54 \mathrm{~cm}(1 \mathrm{in}$.) thick steel cap on the riser shields all gamma rays. The gamma ray dose rate 76.20 ( $30 \mathrm{in}$.) from the riser side is negligible. The calculational uncertainties are a few percent which indicate reliable results. All gamma ray dose rate results are shown in Table 2.1.

\begin{tabular}{|c|c|c|}
\hline \multicolumn{3}{|c|}{ Table 2.1 Gamma Ray Dose Rates Above Tank (mrem/h) } \\
\hline \multicolumn{3}{|c|}{$76.20 \mathrm{~cm}$ ( $30 \mathrm{in}$.) above top of 6 Inch Riser } \\
\hline Dose area & uncovered & $\begin{array}{l}2.54 \mathrm{~cm}(1 \mathrm{in} .) \text { steel } \\
\text { cover }\end{array}$ \\
\hline Within radius of Riser & $0.029(0.0055)$ & $6.3 \mathrm{e}-05(0.0705)$ \\
\hline Outside radius of riser & $2.44 \mathrm{e}-04(0.0087)$ & $4.1 \mathrm{e}-06(0.0295)$ \\
\hline Height above ground & \multicolumn{2}{|c|}{$76.20 \mathrm{~cm}(30 \mathrm{in}$.$) from side of riser$} \\
\hline 0 to $25.4 \mathrm{~cm}$ & \multicolumn{2}{|c|}{$4.74 \mathrm{e}-06(0.1013)$} \\
\hline 25.4 to $50.8 \mathrm{~cm}$ & \multicolumn{2}{|c|}{$6.21 \mathrm{e}-06(0.0601)$} \\
\hline $50.8 \mathrm{to} 76.2 \mathrm{~cm}$ & \multicolumn{2}{|c|}{$701 \mathrm{e}-06000696$} \\
\hline
\end{tabular}


HNF-3361, Rev.0

\subsection{Gamma Ray Dose Rates at Side of Tank}

Gamma ray dose rates were calculated for regions off of a side of the tank. This calculation assumed air in place of the dirt outside the tank. The dose rates were calculated at the outside surface and 1 meter, 2 meters, and 3 meters from the side. Elevations relative to the waste in the tank were varied. The results are presented in Table 2.2 .

\begin{tabular}{|c|c|c|c|c|}
\hline \multicolumn{5}{|c|}{ Table 2.2 Gamma Ray Dose Rates off Tank Side $(\mathrm{mrem} / \mathrm{h})$} \\
\hline $\begin{array}{l}\text { Location relative } \\
\text { to tank waste }\end{array}$ & Surface & 1 meter & 2 meters & 3 meters \\
\hline centered & $2.2 \mathrm{e}-03(0.0199)$ & $1.4 \mathrm{e}-03(0.0129)$ & $8.6 \mathrm{e}-04(0.0154)$ & $5.6 \mathrm{e}-04(0.0185)$ \\
\hline $\begin{array}{c}0 \text { to } 2 \mathrm{ft} \text { above } \\
\text { top }\end{array}$ & $9.5 \mathrm{e}-04(0.0386)$ & $9.2 \mathrm{e}-04(0.0170)$ & $7.3 \mathrm{e}-04(0.0301)$ & $5.3 \mathrm{e}-04(0.0205)$ \\
\hline $\begin{array}{l}6 \text { to } 8 \mathrm{ft} \text { above } \\
\end{array}$ & $4.2 \mathrm{e}-04(0.0655)$ & $3.7 \mathrm{e}-04(0.0349)$ & $3.2 \mathrm{e}-04(0.0329)$ & $2.8 \mathrm{e}-04(0.0332)$ \\
\hline
\end{tabular}

\subsection{Neutron Dose Rates Above Tank}

The neutron dose rates above and around the 6-inch riser are all very small. These dose rate are with the riser uncovered. Covering the riser would have no effect since $2.54 \mathrm{~cm}(1 \mathrm{in}$.) steel is essentially transparent to neutrons. The calculational uncertainties indicate reasonable results. The neutron dose rates are shown in Table 2.3.

\begin{tabular}{||c|c|}
\hline \multicolumn{2}{|c|}{ Table 2.3 Neutron Dose Rates (mrem/h) } \\
\hline $76.20 \mathrm{~cm}$ (30 in.) above top of 6 Inch-Riser & $1.5 \mathrm{e}-04(0.0425)$ \\
\hline Height above ground & $\begin{array}{c}76.20 \mathrm{~cm}(30 \mathrm{in} .) \\
\text { from side of riser }\end{array}$ \\
\hline 0 to $25.4 \mathrm{~cm}$ & $2.5 \mathrm{e}-04(0.0408)$ \\
\hline 25.4 to $50.8 \mathrm{~cm}$ & $2.2 \mathrm{e}-04(0.0417)$ \\
\hline $50.8 \mathrm{to} 76.2 \mathrm{~cm}$ & $22 \mathrm{e}-04(0.0709)$ \\
\hline
\end{tabular}

\subsection{Neutron Dose Rates At Tank Side}

Neutron dose rates were calculated for regions off a side of the tank. This calculation 


\section{HNF-3361, Rev.0}

assumed air in place of the dirt outside the tank. The dose rates were calculated at the outside surface and 1 meter, 2 meters, and 3 meters from the side. Elevations relative to the waste in the tank were varied. The results are presented in Table 2.4.

\begin{tabular}{||l|c|c|c|c||}
\hline \multicolumn{5}{|c|}{ Table 2.4 Neutron Dose Rates off Tank Side (mrem/h) } \\
\hline \begin{tabular}{l}
\hline $\begin{array}{l}\text { ocation relative } \\
\text { to tank waste }\end{array}$ \\
\hline centered
\end{tabular} & $6.8 \mathrm{e}-03(0.0167)$ & $3.8 \mathrm{e}-03(0.0193)$ & $2.1 \mathrm{e}-03(0.0245)$ & $1.3 \mathrm{e}-03(0.0355)$ \\
\hline $\begin{array}{l}0 \text { to } 2 \mathrm{ft} \text { above } \\
\text { op }\end{array}$ & $3.1 \mathrm{e}-03(0.0371)$ & $2.6 \mathrm{e}-03(0.0210)$ & $1.7 \mathrm{e}-03(0.0278)$ & $1.2 \mathrm{e}-03(0.0363)$ \\
\hline $\begin{array}{l}6 \text { to } 8 \mathrm{ft} \text { above } \\
\text { n }\end{array}$ & $1.2 \mathrm{e}-03(0.0683)$ & $1.1 \mathrm{e}-03(0.0607)$ & $9.3 \mathrm{e}-04(0.0769)$ & $6.7 \mathrm{e}-04(0.0471)$ \\
\hline
\end{tabular}

\subsection{MODELING INPUTS}

\subsection{Geometry}

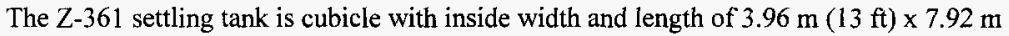
( $26 \mathrm{ft})$, respectively. The tank has an inside height of $5.2 \mathrm{~m}(17 \mathrm{ft})$ at one end and $5.5 \mathrm{~m}(18 \mathrm{ft})$ at the other end. The sloped bottom runs along the $7.92 \mathrm{~m}(26 \mathrm{ft})$ length. The bottom, walls and top

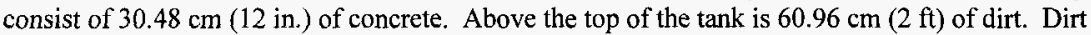
is also modeled below the tank and outside the walls.

A 6-inch riser extends out of the tank top to $76.2 \mathrm{~cm}(30 \mathrm{in}$.) above the ground. The riser is assumed to be a schedule 40 pipe with a $16.828 \mathrm{~cm}(6.625$ in) outer diameter and a $0.711 \mathrm{~cm}$ $(0.28 \mathrm{in}$.) wall thickness. The location of the riser was unclear from the information provided so it was centered along the $3.96 \mathrm{~m}$ (13 ft) width and slightly off center along the $7.92 \mathrm{~m}(26 \mathrm{ft})$ length.

The top of the sludge was modeled as being $2.9 \mathrm{~m}(9.5 \mathrm{ft})$ from the inside top of the tank, for an average depth of $2.44 \mathrm{~m}(8 \mathrm{ft})$.

Tank dimensions were obtained from References 1 and 2 and drawing no. H-2-16460 (Reference 7). Dimensions are shown in Figure 3.1 from Reference 2.

An MCNP (Reference 8) plot of the tank geometry is provided in Figure 3.2. 
HNF-3361, Rev.0

Figure 3.1 Settling Tank Z-361

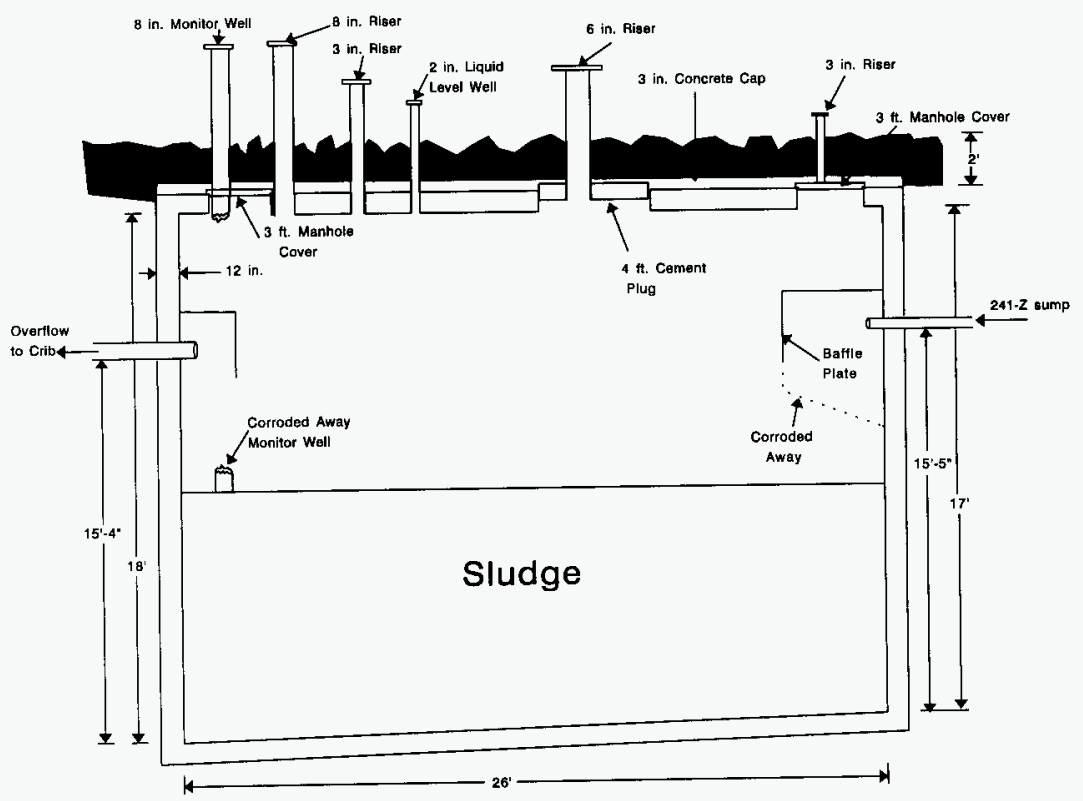


HNF-3361, Rev.0

Figure 3.2 MCNP Plot of Settling Tank Z-361

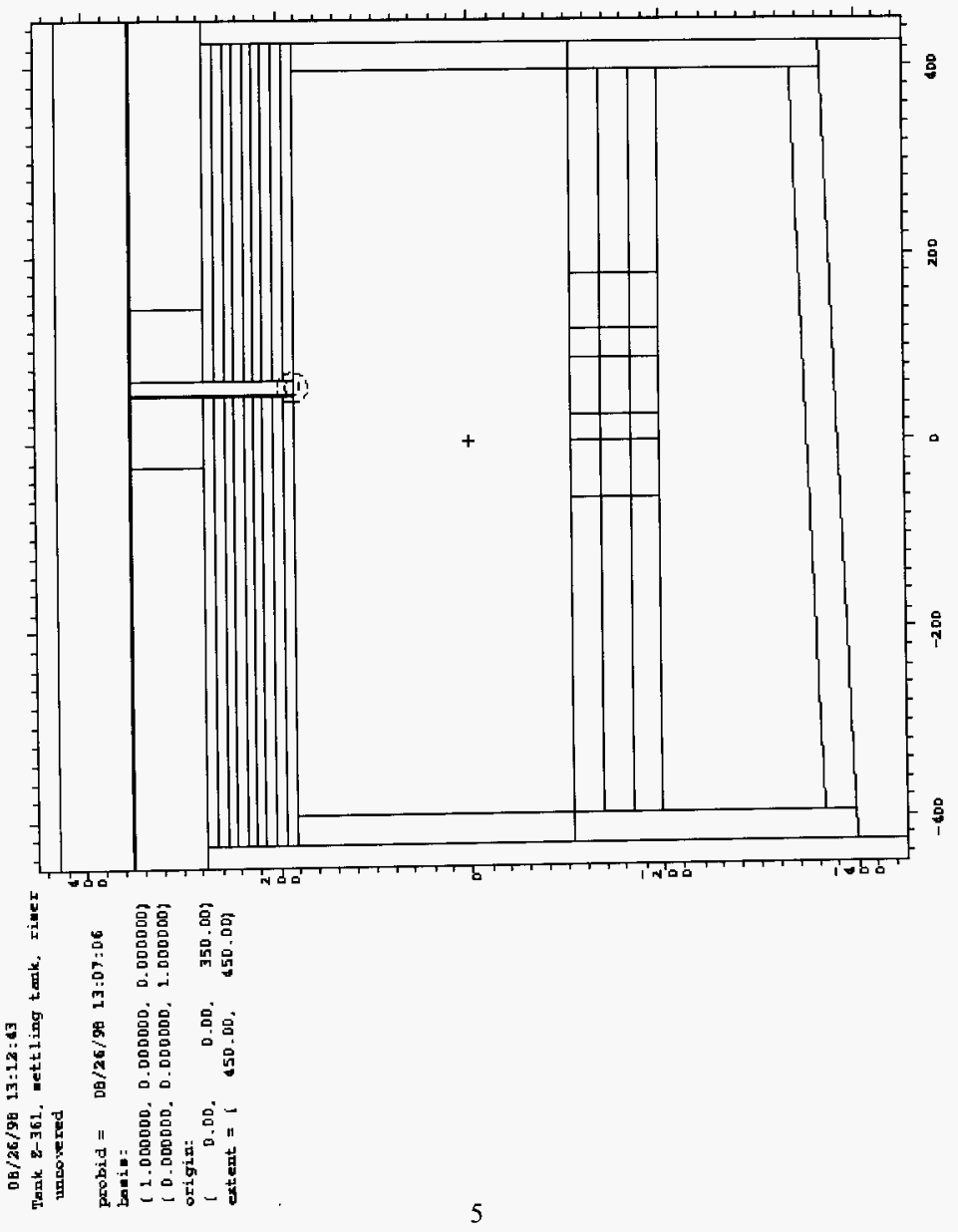


HNF-3361, Rev.0

\subsection{Materials}

The sludge material has a density of $1.14 \mathrm{~g} / \mathrm{cm}^{3}$ for a total volume of 75,000 liters and contains $30 \mathrm{~kg}$ of plutonium. Sludge composition is from reference 1 . Ordinary concrete was used for the tank top, bottom and sides at a density of $2.258 \mathrm{~g} / \mathrm{cm}^{3}$. Hanford dirt has a density of $1.67 \mathrm{~g} / \mathrm{cm}^{3}$. Material compositions as input into MCNP are given in Table 3.1 . The table provides the material constituents in $\mathrm{g} / \mathrm{cm}^{3}$ which were normalized by MCNP to the material density.

\begin{tabular}{|c|c|c|c|c|}
\hline \multicolumn{5}{|c|}{ Table 3.T Material Composition $\left(\mathrm{g} / \mathrm{cm}^{3}\right)$} \\
\hline Element & source $^{\mathrm{a}}$ & stcel & concrete & dirt \\
\hline aluminum & 0.304 & - & 0.0607 & 0.08326 \\
\hline calcium & 0.460 & - & 0.1306 & 0.0717 \\
\hline iron & 0.5622 & 5.1009 & 0.0788 & 0.1091 \\
\hline sodium & 0.0405 & - & 0.0182 & 0.02022 \\
\hline silicon & 0.0104 & 0.0587 & 0.2157 & 0.2782 \\
\hline oxygen & 0.200 & - & 0.4407 & 0.511 \\
\hline hydrogen & 0.060 & - & 0.0031 & - \\
\hline carbon & 0.0872 & 0.0023 & - & - \\
\hline chlorine & 0.0342 & - & - & - \\
\hline fluorine & 0.0039 & - & - & - \\
\hline chromium & - & 1.566 & - & - \\
\hline nickel & - & 0.9396 & - & - \\
\hline manganese & - & 0.1566 & - & 0.001781 \\
\hline phosphorus & - & 0.0035 & 0.0009 & 0.0024 \\
\hline magnesium & - & - & 0.0376 & 0.03142 \\
\hline sulfur & - & 0.0023 & 0.0009 & $=$ \\
\hline krypton & - & - & 0.0066 & - \\
\hline titanium & - & - & 0.0049 & 0.01655 \\
\hline potassium & & & & 001155 \\
\hline
\end{tabular}

a) source composition (sludge) is from reference 1 . 
difficult to achieve in this particular problem. This is due to the fact that the dose region of interest is located far from the source and the particles must pass through a small riser. As a result, particle sampling efficiency is poor. A powerful but demanding method called DXTRAN Sphere was used, under the guidance of Dr. Lee Carter, to overcome this difficulty and achieve credible results. The details of his sample model, review comments and directions throughout the task are included in Appendix B. 
HNF-3361, Rev.0

\subsection{REFERENCES}

1. Engineering Study of the Criticality Issues Associated with Hanford Tank 241-Z-361, HNF-2012, Rev. 0, E.J. Lipke, C.A. Rogers, E.M. Miller, Prepared by the PHMC Companies for the Department of Energy, December 1997.

2. Consequence Analysis-Hydrogen Explosion in the Z-361 Settling Tank, HNF-SD-CP-CN004, Rev.0, B.W. Hall, Fluor Daniel Northwest, September 1997.

3. Radiological Dose Assessment for Vault Storage Concepts, HNF-SD-W460-ER-001, Rev. 0, R.F. Richard, Fluor Daniel Northwest, February 1997.

4. Certification of MCNP Version 4 A for WHC Computer Platforms, L.L. Carter, WHCMP-SD-30001, Rev. 5, Westinghouse Hanford Company, Richland Washington, May 1995.

5. Conversion of ORIGEN2 to SUN Workstations, F.A. Schmittroth, WHC-SD-NR-SWD006, Westinghouse Hanford Company, Richland, Washington, September 1993.

6. American Nuclear Society, "Neutron and Gamma-ray Fluence-to-Dose Factors", ANSI/ANS-6.1.1-1991.

7. Waste Disposal Facilities 241-Z-361 Settling Tank Arrgt. \& Details, Drawing No. H-216460.

8. Breismeister, J. F., 1993, Editor, MCNP - A General Monte Carlo N-Particle Transport Code, Version 4A, LA-12625, Los Alamos National Laboratory, Los Alamos, New Mexico. 
HNF-3361, Rev.0

\section{APPENDIX A \\ MCNP Input Files}

This appendix contains the MCNP input files used to generate the dose rates reported in this document. Each input file is identified by name and the dose rates calculated are identified.

File name: ilpo361 - Used to calculate the gamma ray dose rates above and around the open riser.

Tank Z-361, settling tank, riser uncovered, ilpo361

c photon dose

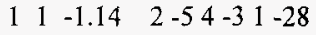

$24-2.258 \quad 2-5-34-17$

$34-2.258-28-54-67$

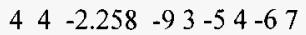

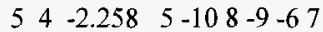

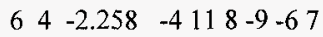

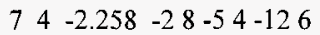

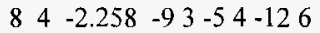

$94-2.258 \quad 5-108-9-126$

$104-2.258 \quad 8-911-46-12$

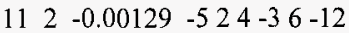

$124 \quad-2.258 \quad 8-10-911 \quad 1434-13$

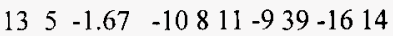

$\begin{array}{llllll}16 & 2 & -0.00129 & -15 & 12 & -17\end{array}$

$\begin{array}{lllllllll}17 & 5 & -1.67 & 20 & -8 & -10 & 11 & 22 & -16\end{array}$

$185-1.67-219-101122-16$

$\begin{array}{lllllllll}19 & 5 & -1.67 & 20 & 10 & -18 & -21 & -1622\end{array}$

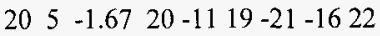

$215-1.67-98-1011-722$

$\begin{array}{lllllll}22 & 3 & -7.86 & 15 & -14 & -17 & 12\end{array}$

$\begin{array}{lllllll}23 & 2 & -0.00129 & 14 & -23 & 16 & -17\end{array}$

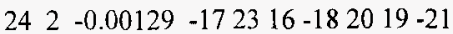

$\begin{array}{lllllllll}25 & 2 & -0.00129 & 20 & -18 & -21 & 19 & 32 & -24\end{array}$

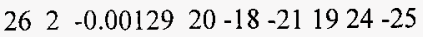

$270(((((18)):(-19)):(21-1819)):(-1819-20))):$ $(20-18-21 \quad 1925)):(20-18-21 \quad 19-22)$

$28 \quad 1-1.14-626-40$

$\begin{array}{llllll}29 & 1 & -1.14 & -26 & 27 & -40\end{array}$

$\begin{array}{lllllll}30 & 1 & -1.14 & -27 & 28 & -40\end{array}$

$\begin{array}{lllllll}31 & 1 & -1.14 & -6 & 26 & 40 & -41\end{array}$ 
HNF-3361, Rev.0

$\begin{array}{lllllll}32 & 1 & -1.14 & -26 & 27 & 40 & -41\end{array}$

$\begin{array}{lllllll}33 & 1 & -1.14 & -27 & 28 & 40 & -41\end{array}$

$\begin{array}{lllllll}34 & 1 & -1.14 & -6 & 26 & 41 & -42\end{array}$

$\begin{array}{lllllllll}35 & 1 & -1.14 & -26 & 27 & 41 & -42\end{array}$

$\begin{array}{llllllllll}36 & 1 & -1.14 & -27 & 28 & 41 & -42\end{array}$

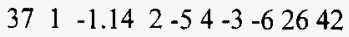

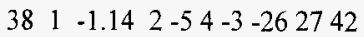

$\begin{array}{llllllllll}39 & 1 & -1.14 & 2 & -5 & 4 & -3 & -27 & 28 & 42\end{array}$

$\begin{array}{llllllllll}40 & 2 & -0.00129 & 20 & -18 & -21 & 19 & 17 & -32 & 14\end{array}$

$\begin{array}{llllll}41 & 2 & -0.00129 & 17 & -32 & -14\end{array}$

$\begin{array}{llllllllll}42 & 4 & -2.258 & 8 & -10 & -9 & 11 & 14 & 12 & -33\end{array}$

$\begin{array}{llllllllll}43 & 4 & -2.258 & 8 & -10 & -9 & 11 & 14 & 33 & -34\end{array}$

$\begin{array}{llllllllll}44 & 5 & -1.67 & -10 & 8 & 11 & -9 & 14 & 13 & -35\end{array}$

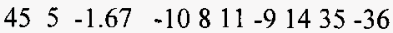

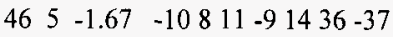

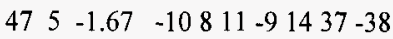

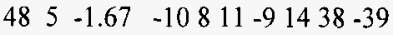

$\begin{array}{cccccc}1 & \mathrm{p}-3.8460000 \mathrm{e}-02 & 0.0000000 & 0.9615400 & 0.0000000 \\ 2 & \mathrm{px} & -396.24000 & & & \\ 3 & \mathrm{px} & 396.24000 & & & \\ 4 & \mathrm{py} & -198.12000 & & & \\ 5 & \mathrm{py} & 198.12000 & & & \\ 6 & \mathrm{pz} & 243.84000 & & & \\ 7 & \mathrm{p}-3.8460000 \mathrm{e}-02 & 0.0000000 & 0.9615400 & -30.480000 \\ 8 & \mathrm{px} & -426.72000 & & & \\ 9 & \mathrm{px} & 426.74000 & & & \\ 10 & \mathrm{py} & 228.60000 & & & \\ 11 & \mathrm{py} & -228.60000 & & & \\ 12 & \mathrm{pz} & 533.40000 & & & \\ 13 & \mathrm{pz} & 563.88000 & & & \\ 14 & \mathrm{c} / \mathrm{z} & 59.436000 & 0.0000000 & 8.4137500 & \\ 15 & \mathrm{c} / \mathrm{z} & 59.436000 & 0.0000000 & 7.7025500 & \\ 16 & \mathrm{pz} & 624.84000 & & & \\ 17 & \mathrm{pz} & 701.04000 & & & \\ 18 & \mathrm{py} & 1752.6000 & & & \\ 19 & \mathrm{py} & -1752.6000 & & & \\ 20 & \mathrm{px} & -1950.7200 & & & \\ 21 & \mathrm{px} & 1950.7200 & & & \\ 22 & \mathrm{pz} & -1600.0000 & & & \\ 23 & \mathrm{c} / \mathrm{z} & 59.436000 & 0.0000000 & 84.613750 & \end{array}$


HNF-3361, Rev.0

\begin{tabular}{|c|c|c|c|c|}
\hline 24 & $\mathrm{pz}$ & \multicolumn{3}{|c|}{777.24000} \\
\hline 25 & $\mathrm{pz}$ & 2301.240 & & \\
\hline 26 & $\mathrm{pz}$ & 213.3600 & & \\
\hline 27 & $\mathrm{pz}$ & 182.880 & & \\
\hline 28 & $\mathrm{pz}$ & 152.400 & & \\
\hline 32 & $\mathrm{pz}$ & 703.550 & & \\
\hline 33 & $\mathrm{pz}$ & 543.56 & & \\
\hline 34 & $\mathrm{pz}$ & 553.72 & & \\
\hline 35 & $\mathrm{pz}$ & 574.04 & & \\
\hline 36 & $\mathrm{pz}$ & 584.20 & & \\
\hline 37 & $\mathrm{pz}$ & 594.36 & & \\
\hline 38 & $\mathrm{pz}$ & 604.52 & & \\
\hline 39 & $\mathrm{pz}$ & 614.68 & & \\
\hline 40 & $\mathrm{c} / \mathrm{z}$ & 59.436 & 0.000 & 30.00 \\
\hline 41 & $\mathrm{c} / \mathrm{z}$ & 59.436 & 0.000 & 60.00 \\
\hline 42 & $c / z$ & 59.436 & 0.000 & 120.00 \\
\hline 50 & $\mathrm{pz}$ & 650.24 & & \\
\hline 51 & $\mathrm{pz}$ & 675.64 & & \\
\hline
\end{tabular}

mode $\mathrm{p}$

m1 13000.01p-0.304 20000.01p -0.460 26000.01p-0.5622 \$source 1.14g/cc $11000.01 \mathrm{p}-0.040514000 .01 \mathrm{p}-0.01048000 .01 \mathrm{p}-0.200$

$1000.01 \mathrm{p}-0.0606000 .01 \mathrm{p}-0.087217000 .01 \mathrm{p}-0.0342$

$9000.01 \mathrm{p}-0.0039$

$\mathrm{m} 2 \quad 7000.01 \mathrm{p} 0.790008000 .01 \mathrm{p} 0.21000 \quad \$$ air $0.00129 \mathrm{~g} / \mathrm{cc}$

m3 26000.01p -5.1009 24000.01p-1.566 28000.01p -0.9396\$ steel $7.86 \mathrm{~g} / \mathrm{cc}$

$25000.01 p-0.156614000 .01 p-0.058715000 .01 p-0.0035$

$16000.01 \mathrm{p}-0.00236000 .01 \mathrm{p}-0.0023$

m4 $1000.01 \mathrm{p}-0.00318000 .01 \mathrm{p}-0.440711000 .01 \mathrm{p}-0.0182$ \$ concrete $2.258 \mathrm{~g} / \mathrm{cc}$

$12000.01 \mathrm{p}-0.037613000 .01 \mathrm{p}-0.060714000 .01 \mathrm{p}-0.2157$

$15000.01 \mathrm{p}-0.000916000 .01 \mathrm{p}-0.000936000 .01 \mathrm{p}-0.0066$

$20000.01 \mathrm{p}-0.130622000 .01 \mathrm{p}-0.004926000 .01 \mathrm{p}-0.0788$

m5 $8000.01 \mathrm{p}-0.51114000 .01 \mathrm{p}-0.278220000 .01 \mathrm{p}-0.0717 \$$ dirt $1.67 \mathrm{~g} / \mathrm{cc}$

$26000.01 p-0.109113000 .01 p-0.0832612000 .01 p-0.03142$

$19000.01 \mathrm{p}-0.0115511000 .01 \mathrm{p}-0.0202222000 .01 \mathrm{p}-0.01655$

$25000.01 \mathrm{p}-0.00178115000 .01 \mathrm{p}-0.0024$

imp:p 0 9r $13 \mathrm{r} 0011014 \mathrm{r} 01.25 .065 .25 .0625 .015625 .0625$

$.015625 .0003906300018 \mathrm{r} \quad \$ 1,46$

cut:p j j 00

c source

c source fraction of total sludge volume (76569 I total volume) 
HNF-3361, Rev.0

c wgt $=(2.926 \mathrm{e}+12 \mathrm{p} / \mathrm{kg} \mathrm{pu}) \times 30 \mathrm{~kg} \mathrm{pu} \times(4137 / 76569)=4.743 \mathrm{e}+12$ sdef $w g t=4.743 \mathrm{e}+12 \mathrm{erg}=\mathrm{d} 1$ cel $=\mathrm{d} 2$ pos fcel $\mathrm{d} 3 \mathrm{rad}$ fcel $\mathrm{d} 4 \mathrm{ext} \mathrm{d} 5$ axs 001

sc1 photo spectrum for $1 \mathrm{~kg}$ pu from HNF-SD-W460-ER-001

\# $\quad$ sil sp1 sb1

l d d

$\begin{array}{lll}0.0150 & 1.409 \mathrm{e}+12 & 0.3\end{array}$

$\begin{array}{lll}0.0250 & 9.578 \mathrm{e}+10 \quad 5.0 \mathrm{e}-2\end{array}$

$\begin{array}{lll}0.0375 & 8.626 \mathrm{e}+09 \quad 8.6 \mathrm{e}-3\end{array}$

$\begin{array}{lll}0.0575 & 1.409 \mathrm{e}+12 & 1.409\end{array}$

$0.0850 \quad 1.306 \mathrm{e}+09 \quad 1.3 \mathrm{e}-2$

$0.1250 \quad 1.492 \mathrm{e}+09 \quad 1.5 \mathrm{e}-1$

$0.2250 \quad 3.656 \mathrm{e}+08 \quad 1.0 \mathrm{e}-1$

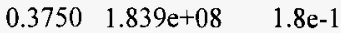

$\begin{array}{lll}0.5750 & 3.078 \mathrm{e}+07 \quad 9.0 \mathrm{e}-2\end{array}$

$0.8500 \quad 9.134 \mathrm{e}+06 \quad 9.1 \mathrm{e}-2$

$1.250 \quad 1.911 \mathrm{e}+05 \quad 6.0 \mathrm{e}-3$

$\begin{array}{lll}1.750 & 8.269 \mathrm{e}+04 & 8.2 \mathrm{e}-3\end{array}$

$2.250 \quad 4.734 \mathrm{e}+04 \quad 1.4 \mathrm{e}-2$

$2.750 \quad 2.718 \mathrm{e}+04 \quad 2.7 \mathrm{e}-2$

$3.500 \quad 2.403 \mathrm{e}+04 \quad 7.0 \mathrm{e}-2$

$5.000 \quad 1.012 \mathrm{e}+04 \quad 1.0 \mathrm{e}-1$

$7.000 \quad 1.143 \mathrm{e}+03 \quad 5.0 \mathrm{e}-2$

$11.00 \quad 1.300 \mathrm{e}+02 \quad 5.0 \mathrm{e}-2$

c $\quad 2.926 \mathrm{e}+12 \mathrm{p} / \mathrm{s} \mathrm{per} \mathrm{kg} \mathrm{pu}$

$\mathrm{sc} 2$ source cells

si2 1282930313233343536

sp2 86.18 86.18 86.18258.54258.54258.54 1034.16 1034.161034.16

sb2 102.5 .6257 .51 .875 .46887 .51 .875 .4688

ds3 159.4360 .0228 .6059 .4360 .0198 .1259 .4360 .0167 .64

59.4360 .0228 .6059 .4360 .0198 .1259 .4360 .0167 .64

59.4360 .0228 .6059 .4360 .0198 .1259 .4360 .0167 .64

ds4 s 414141424242434343

si41 0.30 .

sp41-21 1

$\operatorname{si42} 30.60$.

sp42-21 1

si43 60. 120.

sp43-21 1

si5 -15.2415 .24$

sp5 -210 
HNF-3361, Rev.0

c

print 102040506080100110120126140170

phys:pj 11

prdmp j -2401

dxt:p 59.4360533 .408 .016 .0

dxc:p 09 r $10012 \mathrm{r} 18 \mathrm{r} 04 \mathrm{r} 0004 \mathrm{r}$

ctme 30

c ansi/ans-6.1.1-1991 AP fluence-to-dose,photons $\left(\mathrm{mrem} / \mathrm{hr} /\left(\mathrm{p} / \mathrm{cm}^{* *} 2 / \mathrm{s}\right)\right.$

de0 $\log .01 .015 .02 .03 .04 .05$

.06 .08 .10 .15 .20 .30

.40 .50 .60 .801 .01 .5

2.03 .04 .05 .06 .08 .0

10.12.

df0 $\quad \log 2.232 \mathrm{e}-55.652 \mathrm{e}-5$ 8.568e-5 1.184e-4 1.314e-4 1.382e-4

$1.440 \mathrm{e}-41.624 \mathrm{e}-41.919 \mathrm{e}-42.797 \mathrm{e}-43.708 \mathrm{e}-45.616 \mathrm{e}-4$

$7.416 \mathrm{e}-4$ 9.144e-4 $1.076 \mathrm{e}-3 \quad 1.379 \mathrm{e}-3 \quad 3.656 \mathrm{e}-3 \quad 2.246 \mathrm{e}-3$

$2.758 \mathrm{e}-3 \quad 3.672 \mathrm{e}-3$ 4.500e-3 5.292e-3 6.012e-3 7.488e-3

$8.892 \mathrm{e}-31.040 \mathrm{e}-2$

fc2 surface tally 30 inches above top of riser

$\mathrm{f} 2$ : 24

fs2 - $14-23$

sd2 222.397222701

e2 0.10 .30 .61 .32 .33 .045 .17 .112

fc 12 surface tally 30 inches from side of riser

f12:p 23

fs $12-50-51$

sd12 135041350413504

el2 0.10 .30 .61 .32 .33 .045 .17 .112 


\section{HNF-3361, Rev.0}

File name: ilpc361 - Used to calculate the gamma ray dose rates above and around the closed riser.

Tank Z-361, settling tank, riser cap, ilpc361

c photon dose

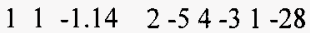

$24-2.258 \quad 2-5-34-17$

$34-2.258-28-54-67$

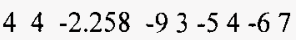

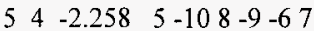

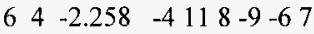

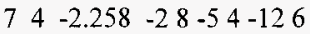

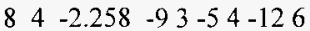

$94-2.258 \quad 5-108-9-126$

$104-2.258 \quad 8-911-46-12$

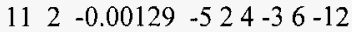

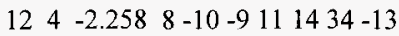

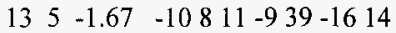

$\begin{array}{llllll}16 & 2 & -0.00129 & -15 & 12 & -17\end{array}$

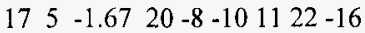

$\begin{array}{lllllllll}18 & 5 & -1.67 & -21 & 9 & -10 & 11 & 22 & -16\end{array}$

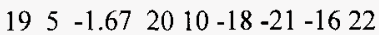

$\begin{array}{lllllll}20 & 5 & -1.67 & 20-11 & 19-21 & -1622\end{array}$

$\begin{array}{llllllllll}21 & 5 & -1.67 & -9 & 8 & -10 & 11 & -7 & 22\end{array}$

$22 \quad 3 \quad-7.86 \quad 15-14-17 \quad 12$

$\begin{array}{lllllll}23 & 2 & -0.00129 & 14 & -23 & 16 & -17\end{array}$

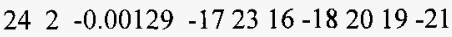

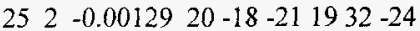

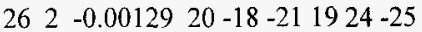

$270(((((18)):(-19))):(21-1819)):(-1819-20))$ : $(20-18-211925)):(20-18-2119-22)$

$\begin{array}{llllll}28 & 1 & -1.14 & -6 & 26 & -40\end{array}$

$\begin{array}{llllll}29 & 1 & -1.14 & -26 & 27 & -40\end{array}$

$\begin{array}{lllllll}30 & 1 & -1.14 & -27 & 28 & -40\end{array}$

$\begin{array}{lllllll}31 & 1 & -1.14 & -6 & 26 & 40 & -41\end{array}$

$\begin{array}{lllllll}32 & 1 & -1.14 & -26 & 27 & 40 & -41\end{array}$

$\begin{array}{lllllll}33 & 1 & -1.14 & -27 & 28 & 40 & -41\end{array}$

$\begin{array}{lllllll}34 & 1 & -1.14 & -6 & 26 & 41 & -42\end{array}$

$\begin{array}{lllllll}35 & 1 & -1.14 & -26 & 27 & 41 & -42\end{array}$

$\begin{array}{llllllllll}36 & 1 & -1.14 & -27 & 28 & 41 & -42\end{array}$

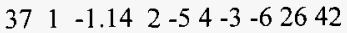


HNF-3361, Rev.0

$38 \quad 1-1.14 \quad 2-54-3-262742$

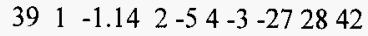

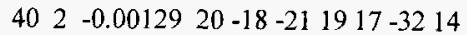

$\begin{array}{llllll}41 & 3 & -7.86 & 17 & -32 & -14\end{array}$

$\begin{array}{llllllllll}42 & 4 & -2.258 & 8 & -10 & -9 & 11 & 14 & 12 & -33\end{array}$

$\begin{array}{llllllllll}43 & 4 & -2.258 & 8 & -10 & -9 & 11 & 14 & 33 & -34\end{array}$

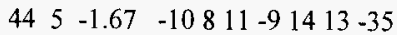

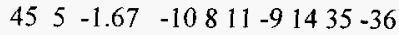

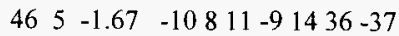

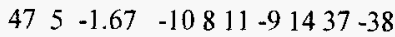

$\begin{array}{llllllllll}48 & 5 & -1.67 & -10 & 8 & 11 & -9 & 14 & 38 & -39\end{array}$

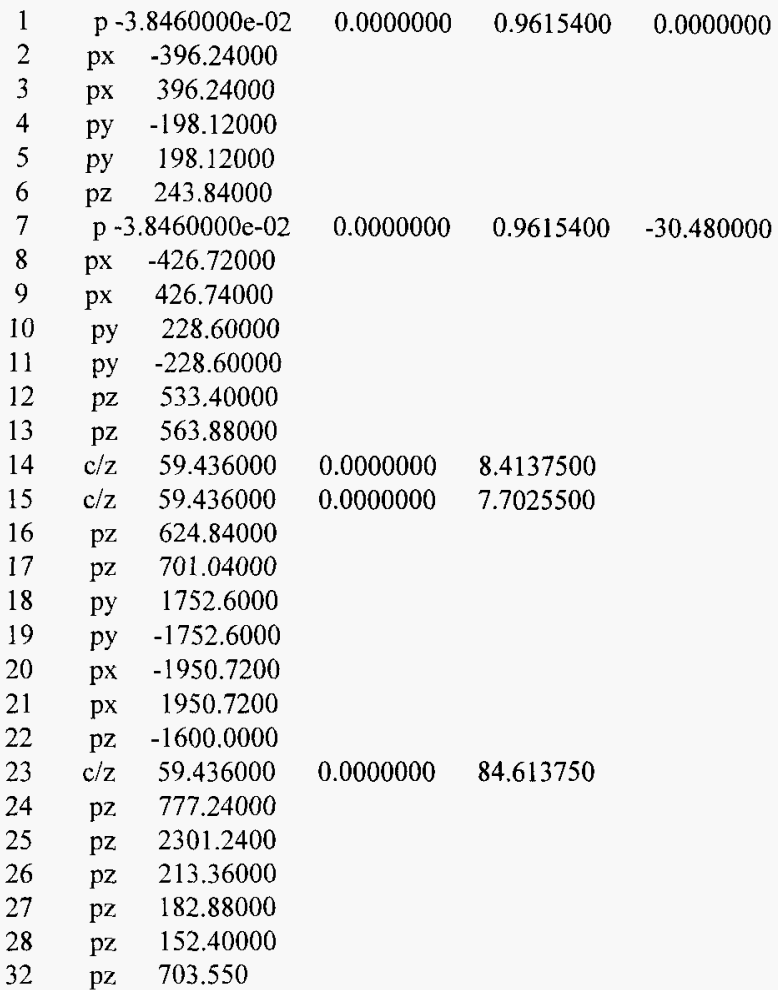


HNF-3361, Rev.0

$\begin{array}{lllll}33 & \mathrm{pz} & 543.56 & & \\ 34 & \mathrm{pz} & 553.72 & & \\ 35 & \mathrm{pz} & 574.04 & & \\ 36 & \mathrm{pz} & 584.20 & & \\ 37 & \mathrm{pz} & 594.36 & & \\ 38 & \mathrm{pz} & 604.52 & & \\ 39 & \mathrm{pz} & 614.68 & & \\ 40 & \mathrm{c} / \mathrm{z} & 59.436 & 0.000 & 30.00 \\ 41 & \mathrm{c} / \mathrm{z} & 59.436 & 0.000 & 60.00 \\ 42 & \mathrm{c} / \mathrm{z} & 59.436 & 0.000 & 120.00 \\ 50 & \mathrm{pz} & 650.24 & & \\ 51 & \mathrm{pz} & 675.64 & & \end{array}$

mode $\mathrm{p}$

m1 13000.01p -0.304 20000.01p-0.460 26000.01p -0.5622 \$source 1.14g/cc $11000.01 \mathrm{p}-0.040514000 .01 \mathrm{p}-0.01048000 .01 \mathrm{p}-0.200$

$1000.01 \mathrm{p}-0.0606000 .01 \mathrm{p}-0.087217000 .01 \mathrm{p}-0.0342$

$9000.01 \mathrm{p}-0.0039$

$\mathrm{m} 2 \quad 7000.01 \mathrm{p} 0.790008000 .01 \mathrm{p} 0.21000 \quad$ \$ air $0.00129 \mathrm{~g} / \mathrm{cc}$

m3 $26000.01 \mathrm{p}-5.100924000 .01 \mathrm{p}-1.56628000 .01 \mathrm{p}-0.9396 \$$ steel $7.86 \mathrm{~g} / \mathrm{cc}$ $25000.01 \mathrm{p}-0.156614000 .01 \mathrm{p}-0.058715000 .01 \mathrm{p}-0.0035$ $16000.01 \mathrm{p}-0.00236000 .01 \mathrm{p}-0.0023$

m4 $1000.01 \mathrm{p}-0.00318000 .01 \mathrm{p}-0.440711000 .01 \mathrm{p}-0.0182 \$$ concrete $2.258 \mathrm{~g} / \mathrm{cc}$ $12000.01 \mathrm{p}-0.037613000 .01 \mathrm{p}-0.060714000 .01 \mathrm{p}-0.2157$ $15000.01 \mathrm{p}-0.000916000 .01 \mathrm{p}-0.000936000 .01 \mathrm{p}-0.0066$ $20000.01 \mathrm{p}-0.130622000 .01 \mathrm{p}-0.004926000 .01 \mathrm{p}-0.0788$

m5 $\quad 8000.01 \mathrm{p}-0.51114000 .01 \mathrm{p}-0.278220000 .01 \mathrm{p}-0.0717 \$$ dirt $1.67 \mathrm{~g} / \mathrm{cc}$ $26000.01 p-0.109113000 .01 p-0.0832612000 .01 p-0.03142$ $19000.01 \mathrm{p}-0.0115511000 .01 \mathrm{p}-0.0202222000 .01 \mathrm{p}-0.01655$ $25000.01 \mathrm{p}-0.00178115000 .01 \mathrm{p}-0.0024$

imp:p 0 9r $13 \mathrm{r} 0011014 \mathrm{r} 01.25 .065 .25 .0625 .015625 .0625$

$.015625 .0003906300018 \mathrm{r} \quad \$ 1,46$ cut:p j j 00

c source

c source fraction of total sludge volume ( 765691 total volume)

c $w g t=(2.926 \mathrm{e}+12 \mathrm{p} / \mathrm{kg} \mathrm{pu}) \times 30 \mathrm{~kg} \mathrm{pu} \times(4137 / 76569)=4.743 \mathrm{e}+12$

sdef $w g t=4.743 \mathrm{e}+12 \mathrm{erg}=\mathrm{d} 1 \mathrm{cel}=\mathrm{d} 2 \mathrm{pos}$ fcel $\mathrm{d} 3 \mathrm{rad}$ fcel $\mathrm{d} 4 \mathrm{ext} \mathrm{d} 5$ axs 001

sc1 photo spectrum for $1 \mathrm{~kg}$ pu from HNF-SD-W460-ER-001

\# $\begin{array}{lll}\text { si } 1 & \text { spl } & \text { sb1 } \\ \text { l } & \text { d } & d\end{array}$ 
HNF-3361, Rev.0

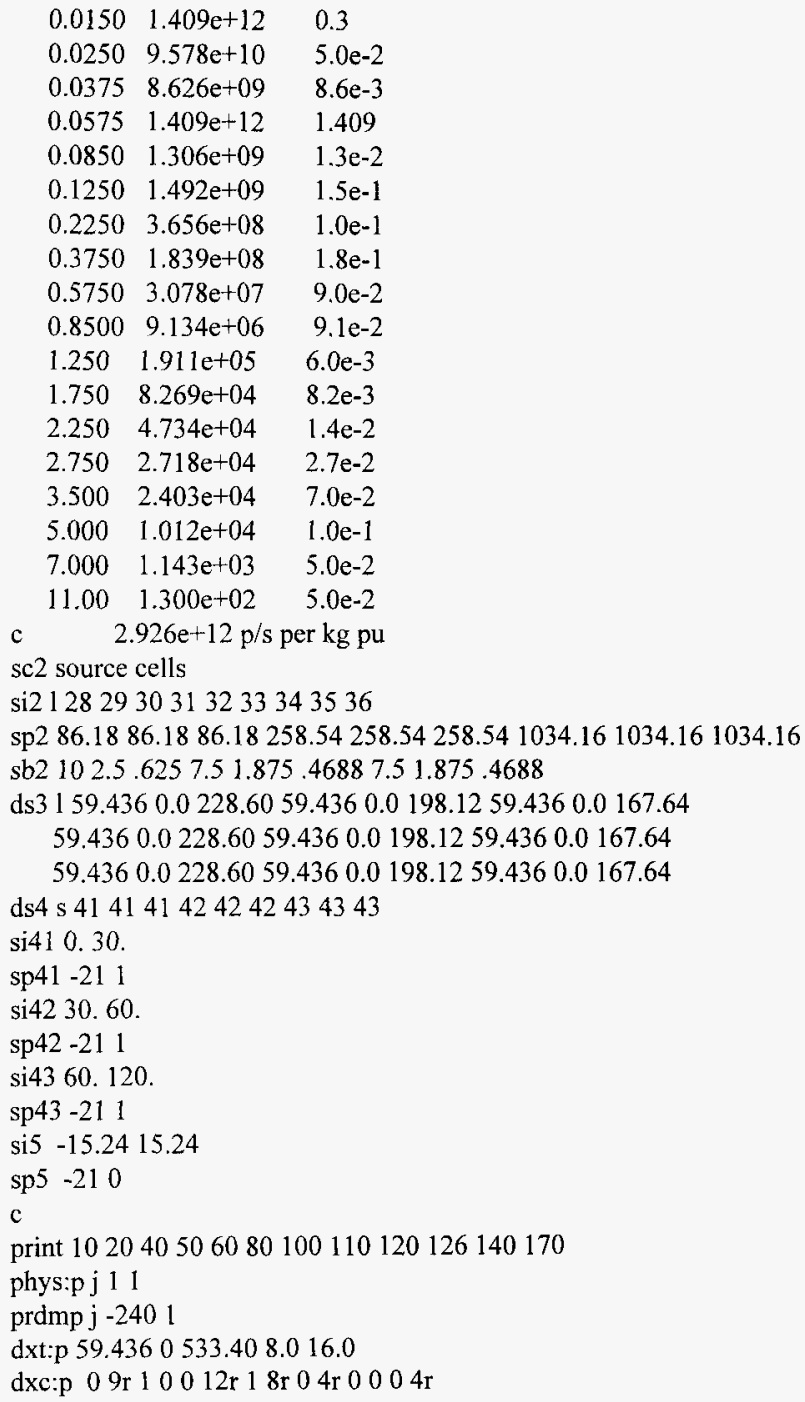




\section{HNF-3361, Rev.0}

ctme 1200

c ansi/ans-6.1.1-1991 AP fluence-to-dose,photons $\left(\mathrm{mrem} / \mathrm{hr} /\left(\mathrm{p} / \mathrm{cm}^{* *} 2 / \mathrm{s}\right)\right.$

de0 $\log .01 .015 .02 .03 .04 .05$

.06 .08 .10 .15 .20 .30

.40 .50 .60 .801 .01 .5

2.03 .04 .05 .06 .08 .0

10. 12.

df0 $\log 2.232 \mathrm{e}-55.652 \mathrm{e}-5 \quad 8.568 \mathrm{e}-5 \quad 1.184 \mathrm{e}-4 \quad 1.314 \mathrm{e}-41.382 \mathrm{e}-4$

$1.440 \mathrm{e}-41.624 \mathrm{e}-41.919 \mathrm{e}-42.797 \mathrm{e}-4$ 3.708e-4 5.616e-4

$7.416 \mathrm{e}-49.144 \mathrm{e}-41.076 \mathrm{e}-31.379 \mathrm{e}-3 \quad 1.656 \mathrm{e}-32.246 \mathrm{e}-3$

$2.758 \mathrm{e}-3$ 3 3.672e-3 4.500e-3 5.292e-3 6.012e-3 7.488e-3

$8.892 \mathrm{e}-31.040 \mathrm{e}-2$

fc2 surface tally 30 inches above top of riser

f2:p 24

fs2 - $14-23$

sd2 222.397222701

e2 0.10 .30 .61 .32 .33 .045 .17 .112

fc1 2 surface tally 30 inches from side of riser

f12:p 23

fs $12-50-51$

sd12 135041350413504

el2 0.10 .30 .61 .32 .33 .045 .17 .112 


\section{HNF-3361, Rev.0}

File name: no361b - Used to calculate the neutron dose rate above and around the open riser.

Tank Z-361, settling tank, riser uncovered, no361b

c include extra cells for importances and dxtran

c neutron dose

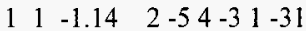

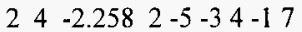

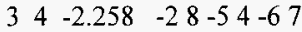

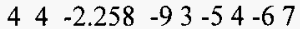

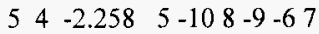

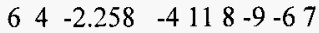

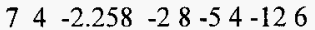

$$
\begin{aligned}
& 84-2.258-93-54-126 \\
& 94-2.2585-108-9-126 \\
& 104-2.258 \quad 8-911-46-12
\end{aligned}
$$

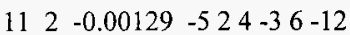

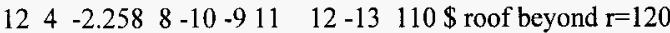

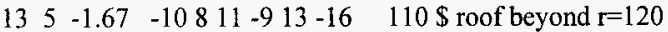

$$
\begin{aligned}
& \begin{array}{llllll}
16 & 2 & -0.00129 & -15 & 12 & -17
\end{array} \\
& 175-1.6720-8-101122-16
\end{aligned}
$$

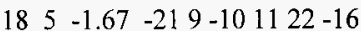

$$
\begin{aligned}
& 195 \quad-1.67 \quad 2010-18-21-1622 \\
& 20 \quad 5-1.67 \quad 20-11 \quad 19-21-1622 \\
& \begin{array}{llllllllll}
21 & 5 & -1.67 & -9 & 8 & -10 & 11 & -7 & 22
\end{array} \\
& \begin{array}{llllllll}
22 & 3 & -7.86 & 15 & -14 & -17 & 12
\end{array} \\
& \begin{array}{lllllll}
23 & 2 & -0.00129 & 14 & -23 & 16 & -17
\end{array}
\end{aligned}
$$

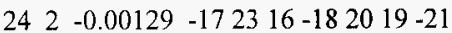

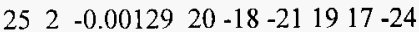

$$
\begin{aligned}
& 26 \quad 2 \quad-0.0012920-18-21 \quad 1924-25 \\
& 27 \quad 0 \quad((((18):(-19))):(21-1819)):(-1819-20)): \\
& (20-18-211925)):(20-18-2119-22) \\
& 28 \quad 1-1.14-626-52-34 \quad 121
\end{aligned}
$$

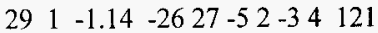

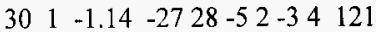

$$
\begin{aligned}
& \begin{array}{lllllllll}
31 & 1 & -1.14 & -28 & 29 & -5 & 2 & -3 & 4
\end{array} \\
& \begin{array}{lllllllll}
32 & 1 & -1.14 & -29 & 30 & -5 & 2 & -3 & 4
\end{array} \\
& \begin{array}{lllllllll}
33 & 1 & -1.14 & -30 & 31 & -5 & 2 & -3 & 4
\end{array} \\
& \text { c additional cells in roof for radius out to } 120 \mathrm{~cm} \text { around riser }
\end{aligned}
$$

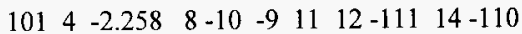

$$
\begin{aligned}
& \begin{array}{llllllllllll}
102 & 4 & -2.258 & 8 & -10 & -9 & 11 & 111 & -13 & 14 & -110
\end{array}
\end{aligned}
$$

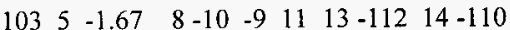


HNF-3361, Rev.0

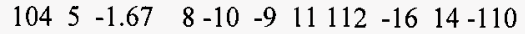

c additional cells in top part of source below riser

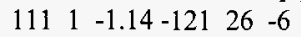

$\begin{array}{lllllll}112 & 1 & -1.14 & -121 & 27 & -26\end{array}$

$\begin{array}{llllll}113 & 1 & -1.14 & -121 & 28 & -27\end{array}$

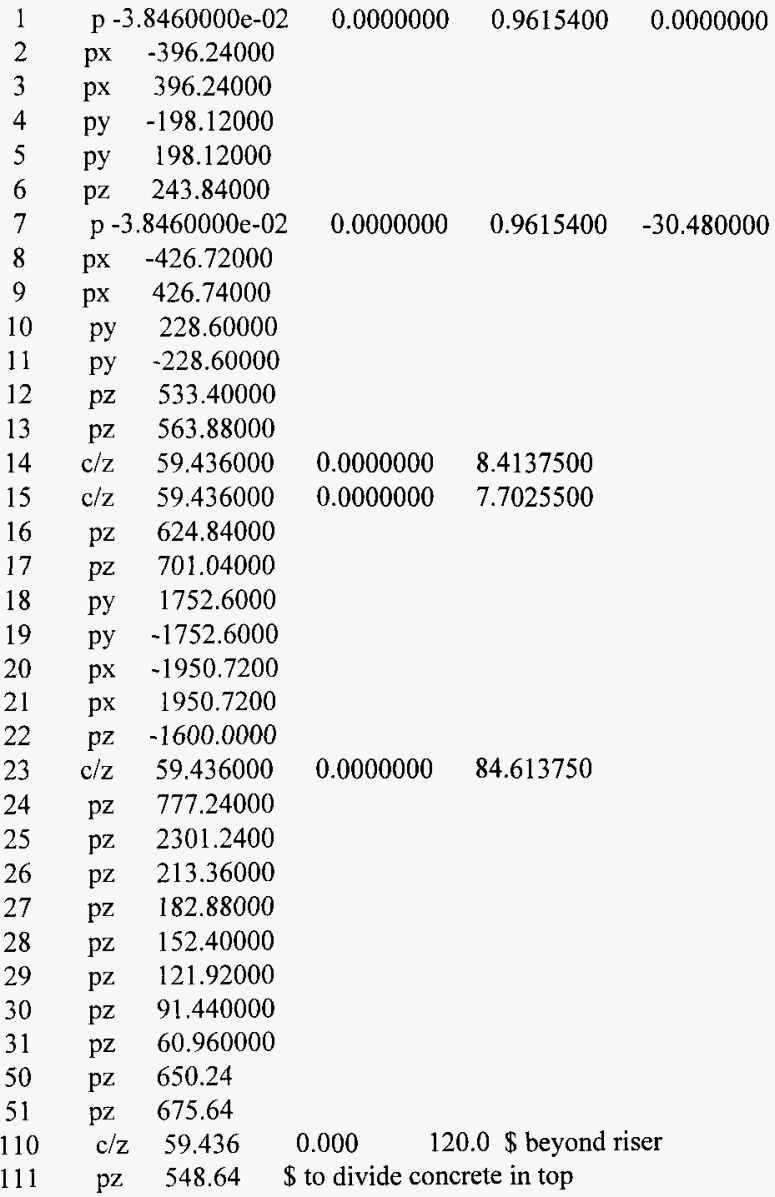


HNF-3361, Rev.0

$112 \quad \mathrm{pz} \quad 594.36 \quad \$$ to divide soil in top

$121 \mathrm{c} / \mathrm{z} \quad 59.436 \quad 0.000 \quad 56.0 \$$ inner source

mode $n$

$\mathrm{m} 1$ 13027.50c $-0.30420000 .50 \mathrm{c}-0.46026000 .55 \mathrm{c}-0.5622$ \$source $1.14 \mathrm{~g} / \mathrm{cc}$

$11023.50 \mathrm{c}-0.040514000 .50 \mathrm{c}-0.01048016 .50 \mathrm{c}-0.200$

$1001.50 \mathrm{c}-0.0606000 .50 \mathrm{c}-0.087217000 .50 \mathrm{c}-0.0342$

$9019.50 \mathrm{c}-0.0039$

$\mathrm{m} 2 \quad 7014.50 \mathrm{c} 0.790008016 .50 \mathrm{c} 0.21000 \quad \$$ air $0.00129 \mathrm{~g} / \mathrm{cc}$

m3 26000.55c -5.1009 24000.50c-1.566 28000.50c -0.9396 \$ steel $7.86 \mathrm{~g} / \mathrm{cc}$

$25055.50 \mathrm{c}-0.156614000 .50 \mathrm{c}-0.058715031 .50 \mathrm{c}-0.0035$

$16032.50 \mathrm{c}-0.00236000 .50 \mathrm{c}-0.0023$

$\mathrm{m} 4 \quad 1001.50 \mathrm{c}-0.00318016 .50 \mathrm{c}-0.440711023 .50 \mathrm{c}-0.0182 \$$ concrete $2.258 \mathrm{~g} / \mathrm{cc}$

$12000.50 \mathrm{c}-0.037613027 .50 \mathrm{c}-0.060714000 .50 \mathrm{c}-0.2157$

$15031.50 \mathrm{c}-0.000916032 .50 \mathrm{c}-0.000936084 .50 \mathrm{c}-0.0066$

$20000.50 c-0.130622000 .50 c-0.004926000 .55 c-0.0788$

m5 $8016.50 \mathrm{c}-0.51114000 .50 \mathrm{c}-0.278220000 .50 \mathrm{c}-0.0717 \$$ dirt $1.67 \mathrm{~g} / \mathrm{cc}$

$26000.55 \mathrm{c}-0.109113027 .50 \mathrm{c}-0.0832612000 .50 \mathrm{c}-0.03142$

$19000.50 \mathrm{c}-0.0115511023 .50 \mathrm{c}-0.0202222000 .50 \mathrm{c}-0.01655$

$25055.50 \mathrm{c}-0.00178115031 .50 \mathrm{c}-0.0024$

imp:n .0104r 103 r $16080640 \quad \$$ to 13

512004 r $10240204805120256025600 \quad$ \$ to 27

$102.5 .625 .15625 .0391 .0391 \quad \$$ to 33

$\begin{array}{llllll}320 & 1280 & 512020480 & 160 & 20 & 2.5\end{array}$

c source

c wgt $=(3.747 \mathrm{e}+05 \mathrm{n} / \mathrm{kg} \mathrm{pu}) \times 30 \mathrm{~kg}$ pu $\times(57427 / 76569)=8.431 \mathrm{e}+06$

sdef wgt $=8.431 \mathrm{e}+06 \mathrm{erg}=\mathrm{d} 1$ cel $=\mathrm{d} 2$ pos $=000 \mathrm{x}$ fcel d3 y fcel d $\mathrm{z}$ fcel d 5

$\mathrm{scl}$ spontaneous fission source spectrum (pu240)

spl -30.7994 .903$

sc2 source cells

si2 1282930313233111112113

sp2 $0.9686252 \mathrm{r} 11110.0313752 \mathrm{r}$

sb2 102.5 .625 .15625 .0391 .03915 .020 .62750 .0784375

ds 3 s $313131313131 \quad 323232$

si31 -396.24396 .24$

sp31 01

si32 3.436115 .436

sp32 001

ds4 s $414141414141 \quad 42 \quad 42 \quad 42$

si41 -198.12 198.12

sp41 01 


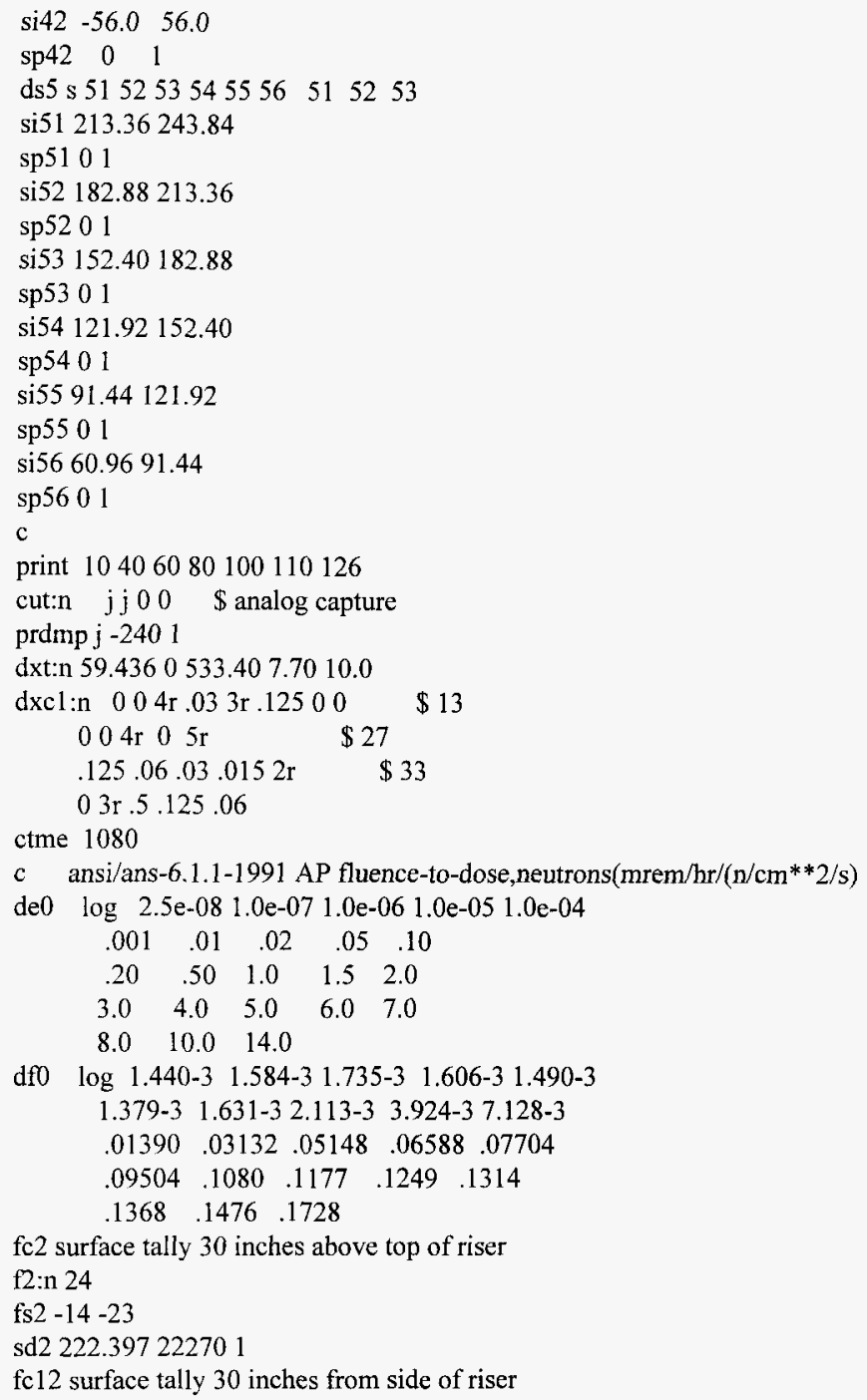

fc2 surface tally 30 inches above top of riser

f $2: \mathrm{n} 24$

fs $2-14-23$

sd2 222.397222701

fc12 surface tally 30 inches from side of riser 
HNF-3361, Rev.0

f12:n 23

fs $12-50-51$

sd12 135041350413504

A-15 


\section{HNF-3361, Rev.0}

File name: ilps $36 \mathrm{a}$ - Used to calculate the gamma ray dose rate off of the tank side.

Tank Z-361, settling tank, riser uncovered, ilps36a

c photon dose at side of tank, point detectors

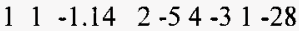

$$
\begin{aligned}
& \begin{array}{llllllll}
2 & 4 & -2.258 & 2 & -5 & -3 & 4 & -1
\end{array}
\end{aligned}
$$

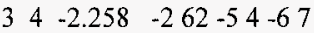

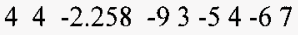

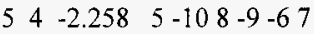

$$
\begin{aligned}
& 64-2.258 \quad-4118-9-67
\end{aligned}
$$

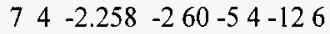

$$
\begin{aligned}
& 84-2.258-93-54-126 \\
& 94-2.258 \quad 5-108-9-126 \\
& 104-2.258 \quad 8-911-46-12
\end{aligned}
$$

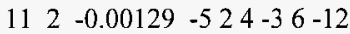

$$
\begin{aligned}
& 12 \quad 4 \quad-2.258 \quad 8-10-911 \quad 1434-13
\end{aligned}
$$

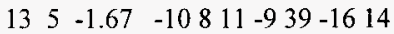

$$
\begin{aligned}
& \begin{array}{llllll}
16 & 2 & -0.00129 & -15 & 12 & -17
\end{array}
\end{aligned}
$$

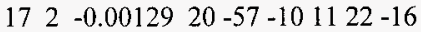

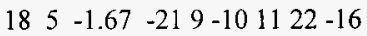

$$
\begin{aligned}
& 195-1.672010-18-21-1622
\end{aligned}
$$

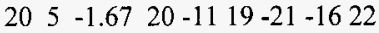

$$
\begin{aligned}
& \begin{array}{lllllllll}
21 & 5 & -1.67 & -9 & 8 & -10 & 11 & -7 & 22
\end{array} \\
& \begin{array}{lllllll}
22 & 3 & -7.86 & 15 & -14 & -17 & 12
\end{array} \\
& \begin{array}{lllllll}
23 & 2 & -0.00129 & 14 & -23 & 16 & -17
\end{array}
\end{aligned}
$$

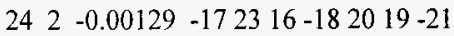

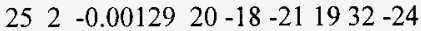

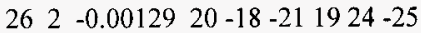

$$
\begin{aligned}
& 270(((((18):(-19)):(21-1819)):(-1819-20)) \text { : } \\
& (20-18-211925)):(20-18-2119-22)
\end{aligned}
$$

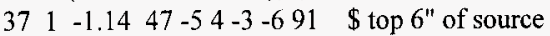

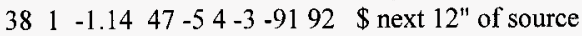

$$
\begin{aligned}
& \begin{array}{lllllllll}
39 & 1 & -1.14 & 47 & -5 & 4 & -3 & -92 & 28
\end{array}
\end{aligned}
$$

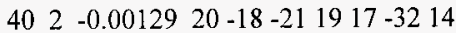

$$
\begin{aligned}
& \begin{array}{llllll}
41 & 2 & -0.00129 & 17 & -32 & -14
\end{array} \\
& \begin{array}{llllllllll}
42 & 4 & -2.258 & 8 & -10 & -9 & 11 & 14 & 12 & -33
\end{array} \\
& \begin{array}{llllllllll}
43 & 4 & -2.258 & 8 & -10 & -9 & 11 & 14 & 33 & -34
\end{array} \\
& \begin{array}{llllllllll}
44 & 5 & -1.67 & -10 & 8 & 11 & -9 & 14 & 13 & -35
\end{array}
\end{aligned}
$$

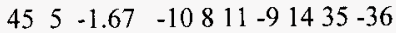

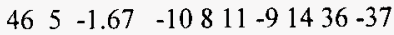

$$
\begin{aligned}
& \begin{array}{llllllllll}
47 & 5 & -1.67 & -10 & 8 & 11 & -9 & 14 & 37 & -38
\end{array}
\end{aligned}
$$




\section{HNF-3361, Rev.0}

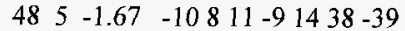

$\begin{array}{llllllllllllll}51 & 1 & -1.14 & 2 & -45 & 4 & -5 & -6 & 26\end{array}$

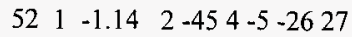

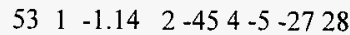

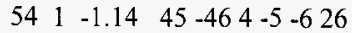

$\begin{array}{lllllllll}55 & 1 & -1.14 & 45 & -46 & 4 & -5 & -26 & 27\end{array}$

$\begin{array}{llllllllll}56 & 1 & -1.14 & 45 & -46 & 4 & -5 & -27 & 28\end{array}$

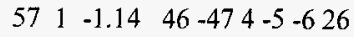

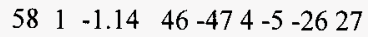

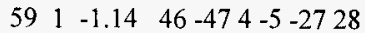

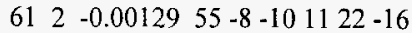

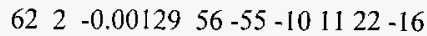

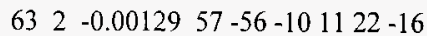

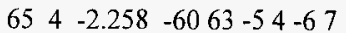

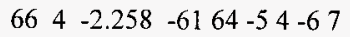

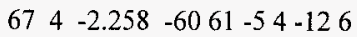

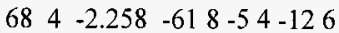

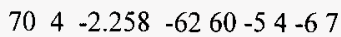

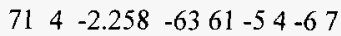

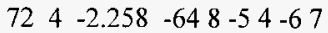

$\begin{array}{cccccc}1 & \mathrm{p}-3.8460000 \mathrm{e}-02 & 0.0000000 & 0.9615400 & 0.0000000 \\ 2 & \mathrm{px} & -396.24000 & & & \\ 3 & \mathrm{px} & 396.24000 & & & \\ 4 & \mathrm{py} & -198.12000 & & & \\ 5 & \mathrm{py} & 198.12000 & & & \\ 6 & \mathrm{pz} & 243.84000 & & & \\ 7 & \mathrm{p}-3.8460000 \mathrm{e}-02 & 0.0000000 & 0.9615400 & -30.480000 \\ 8 & \mathrm{px} & -426.72000 & & & \\ 9 & \mathrm{px} & 426.74000 & & & \\ 10 & \mathrm{py} & 228.60000 & & & \\ 11 & \mathrm{py} & -228.60000 & & & \\ 12 & \mathrm{pz} & 533.40000 & & & \\ 13 & \mathrm{pz} & 563.88000 & & & \\ 14 & \mathrm{c} / \mathrm{z} & 59.436000 & 0.0000000 & 8.4137500 & \\ 15 & \mathrm{c} / \mathrm{z} & 59.436000 & 0.0000000 & 7.7025500 & \\ 16 & \mathrm{pz} & 624.84000 & & & \\ 17 & \mathrm{pz} & 701.04000 & & & \\ 18 & \mathrm{py} & 1752.6000 & & & \\ 19 & \mathrm{py} & -1752.6000 & & & \\ 20 & \mathrm{px} & -1950.7200 & & & \end{array}$


HNF-3361, Rev.0

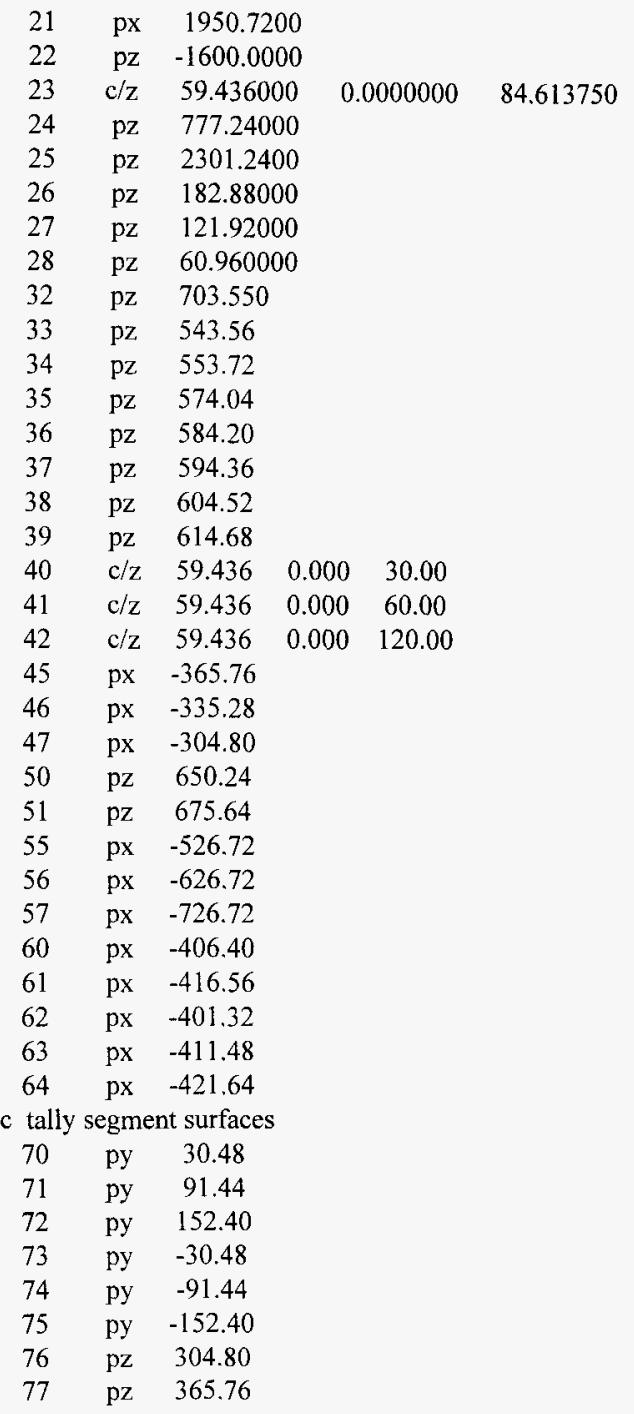


HNF-3361, Rev.0

$\begin{array}{llcl}78 & \mathrm{pz} & 426.72 & \\ 79 & \mathrm{pz} & 487.68 & \\ 80 & \mathrm{pz} & 0.0 & \\ 91 & \mathrm{pz} & 228.60 & \$ 6^{\prime \prime} \text { below top of source } \\ 92 & \mathrm{pz} & 198.12 & \$ 18^{\prime \prime} \text { below top of source }\end{array}$

mode $\mathrm{p}$

$\mathrm{m} 1 \quad 13000.01 \mathrm{p}-0.30420000 .01 \mathrm{p}-0.46026000 .01 \mathrm{p}-0.5622$ \$source $1.14 \mathrm{~g} / \mathrm{cc}$

$11000.01 \mathrm{p}-0.040514000 .01 \mathrm{p}-0.01048000 .01 \mathrm{p}-0.200$

$1000.01 \mathrm{p}-0.0606000 .01 \mathrm{p}-0.087217000 .01 \mathrm{p}-0.0342$

$9000.01 \mathrm{p}-0.0039$

$\mathrm{m} 2 \quad 7000.01 \mathrm{p} 0.790008000 .01 \mathrm{p} 0.21000 \quad \$$ air $0.00129 \mathrm{~g} / \mathrm{cc}$

m3 26000.01p-5.1009 24000.01p-1.566 28000.01p -0.9396 \$ steel $7.86 \mathrm{~g} / \mathrm{cc}$

$25000.01 \mathrm{p}-0.156614000 .01 \mathrm{p}-0.058715000 .01 \mathrm{p}-0.0035$

$16000.01 \mathrm{p}-0.00236000 .01 \mathrm{p}-0.0023$

$\mathrm{m} 4 \quad 1000.01 \mathrm{p}-0.00318000 .01 \mathrm{p}-0.440711000 .01 \mathrm{p}-0.0182 \$$ concrete $2.258 \mathrm{~g} / \mathrm{cc}$

$12000.01 \mathrm{p}-0.037613000 .01 \mathrm{p}-0.060714000 .01 \mathrm{p}-0.2157$

$15000.01 \mathrm{p}-0.000916000 .01 \mathrm{p}-0.000936000 .01 \mathrm{p}-0.0066$

$20000.01 \mathrm{p}-0.130622000 .01 \mathrm{p}-0.004926000 .01 \mathrm{p}-0.0788$

m5 $8000.01 \mathrm{p}-0.51114000 .01 \mathrm{p}-0.278220000 .01 \mathrm{p}-0.0717 \$$ dirt $1.67 \mathrm{~g} / \mathrm{cc}$

$26000.01 \mathrm{p}-0.109113000 .01 \mathrm{p}-0.0832612000 .01 \mathrm{p}-0.03142$

$19000.01 \mathrm{p}-0.0115511000 .01 \mathrm{p}-0.0202222000 .01 \mathrm{p}-0.01655$

$25000.01 \mathrm{p}-0.00178115000 .01 \mathrm{p}-0.0024$

imp:p $101602 \mathrm{r} 32.52 \mathrm{r} 102 \mathrm{r} 51209 \mathrm{r} 1.12502 \mathrm{r} .505 \mathrm{r} \$$ cell 1 to 48

l 2r.5.25.25.25.0625 1r $5122 \mathrm{r} 6425612851232128512$

c imp:p 10102 r 202 r 102 r 32030 r 12 r.252r.06252r32 2r 4168

c 32

c $28,32 \quad \$ 1,65$

c source

c source fraction of total sludge volume (76569 1 total volume)

c $w g t=(2.926 \mathrm{e}+12 \mathrm{p} / \mathrm{kg} \mathrm{pu}) \times 30 \mathrm{~kg}$ pu $\times(19326 / 76569)=22.16 \mathrm{e}+12$

sdef wgt $=22.16 \mathrm{e}+12 \mathrm{erg}=\mathrm{d} 1 \mathrm{cel}=\mathrm{d} 2$ pos $000 \mathrm{x}$ fcel d3 y fcel d $4 \mathrm{z}$ fcel d 5

$\mathrm{scl}$ photo spectrum for $1 \mathrm{~kg}$ pu from HNF-SD-W460-ER-001

\# sil $_{\mathrm{l}}^{\mathrm{sp} 1} \mathrm{~d}$ d ${ }^{\mathrm{sb} 1}$

$\begin{array}{lll}0.0150 & 1.409 \mathrm{e}+12 \quad 1.4 \mathrm{e}-3\end{array}$

$\begin{array}{lll}0.0250 & 9.578 \mathrm{e}+10 \quad 2.0 \mathrm{e}-3\end{array}$

$\begin{array}{lll}0.0375 & 8.626 \mathrm{e}+09 \quad 4.6 \mathrm{e}-4\end{array}$

$0.0575 \quad 1.409 \mathrm{e}+12 \quad 1.4 \mathrm{e}-1$

$\begin{array}{lll}0.0850 & 1.306 \mathrm{e}+09 & 1.3 \mathrm{e}-2\end{array}$

$\begin{array}{lll}0.1250 & 1.492 \mathrm{e}+09 & 1.5 \mathrm{e}-1\end{array}$ 
HNF-3361, Rev.0

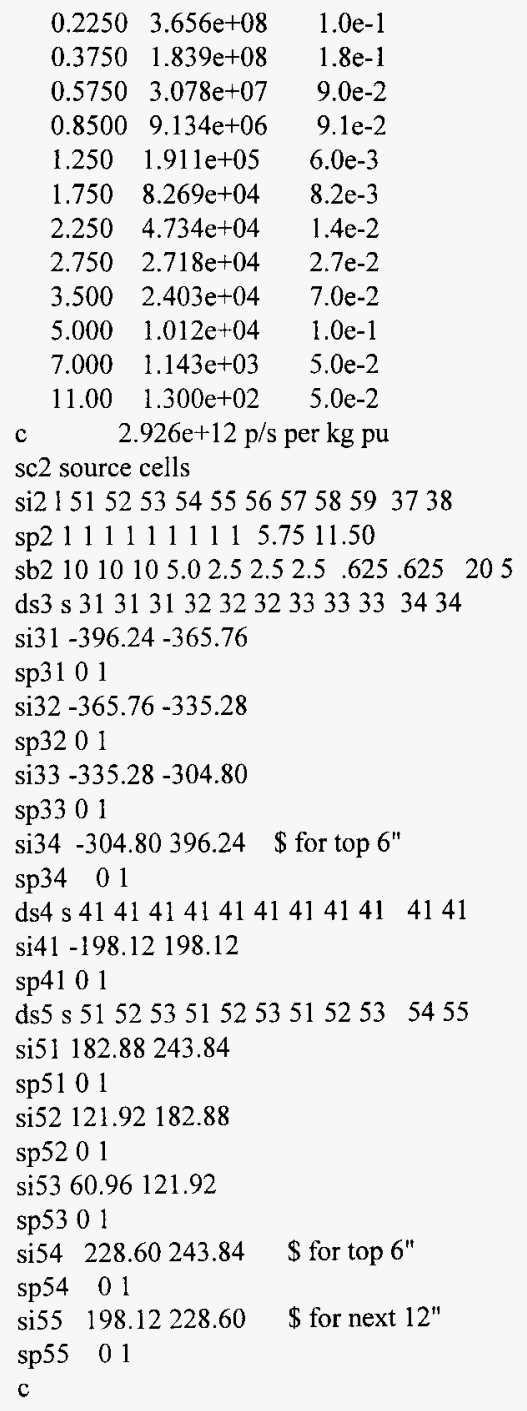


HNF-3361, Rev.0

print 102040506080100110120126140170

phys:p j 11

prdmp j -2401

ctme 30

c ansi/ans-6.1.1-1991 AP fluence-to-dose,photons $\left(\mathrm{mrem} / \mathrm{hr} /\left(\mathrm{p} / \mathrm{cm}^{* *} 2 / \mathrm{s}\right)\right.$

de0 $\log .01 .015 .02 .03 .04 .05$

.06 .08 .10 .15 .20 .30

.40 .50 .60 .801 .01 .5

2.03 .04 .05 .06 .08 .0

10. 12 .

dfo $\quad \log 2.232 \mathrm{e}-55.652 \mathrm{e}-58.568 \mathrm{e}-5 \quad 1.184 \mathrm{e}-4$ 1.314e-4 1.382e-4

$1.440 \mathrm{e}-41.624 \mathrm{e}-41.919 \mathrm{e}-42.797 \mathrm{e}-4$ 3.708e-4 5.616e-4

$7.416 \mathrm{e}-4$ 9.144e-4 1.076e-3 $1.379 \mathrm{e}-3$ 1.656e-3 $2.246 \mathrm{e}-3$

$2.758 \mathrm{e}-3 \quad 3.672 \mathrm{e}-3$ 4.500e-3 $5.292 \mathrm{e}-3 \quad 6.012 \mathrm{e}-3 \quad 7.488 \mathrm{e}-3$

$8.892 \mathrm{e}-31.040 \mathrm{e}-2$

fc2 tank side surface, near bottom away from source cells

f2:p 8

fs2 $-7370-8028$

sd2 11113716.12

fc12 tank side surface, centered on source cells

f12:p 8

fs $12-7370-2726$

sd12 111113716.12

fc22 tank side surface, at source top elevation

f22:p 8

fs $22-7370-676$

$\operatorname{sd} 22111113716.12$

fc32 tank side surface, $7 \mathrm{ft}$ above source top elevation

f32:p 8

fs $32-7370-7879$

sd32 111113716.12

fc42 $1 \mathrm{~m}$ from tank side surface, near bottom away from source cells

f42:p 55

fs $42-7370-8028$

sd42 11113716.12

fc52 $1 \mathrm{~m}$ from tank side surface, centered on source cells

f52:p 55

fs52 $-7370-2726$

sd52 11113716.12

fe62 $1 \mathrm{~m}$ from tank side surface, at source top elevation

f62:p 55 
HNF-3361, Rev.0

fs62 $-7370-676$

sd62 111113716.12

fc72 $1 \mathrm{~m}$ from tank side surface, $7 \mathrm{ft}$ above source top elevation

f72:p 55

fs $72-7370-7879$

sd72 111113716.12

fc82 $2 \mathrm{~m}$ from tank side surface, near bottom away from source cells

f82:p 56

fs $82 \quad-7370-8028$

sd82 111113716.12

fc92 $2 \mathrm{~m}$ from tank side surface, centered on source cells

f92:p 56

fs $92-7370-2726$

sd92 111113716.12

fc102 $2 \mathrm{~m}$ from tank side surface, at source top elevation

f102:p 56

fs $102-7370-676$

sd102 111113716.12

fc112 $2 \mathrm{~m}$ from tank side surface, $7 \mathrm{ft}$ above source top elevation

f112:p 56

fs112 $-7370-7879$

sd112 111113716.12

fc122 $3 \mathrm{~m}$ from tank side surface, near bottom away from source cells

f122:p 57

fs $122-7370-8028$

sd122 111113716.12

fc132 $3 \mathrm{~m}$ from tank side surface, centered on source cells

f132:p 57

fs132 $-7370-2726$

sd132 111113716.12

fc142 $3 \mathrm{~m}$ from tank side surface, at source top elevation

fl $42: p 57$

fs $142-7370-676$

sd142 111113716.12

fc152 $3 \mathrm{~m}$ from tank side surface, $7 \mathrm{ft}$ above source top elevation

f152:p 57

fs $152-7370-7879$

$\operatorname{sd} 152 \quad 111113716.12$

e 0.10 .30 .61 .32 .33 .045 .17 .112 


\section{HNF-3361, Rev.0}

File name: ns361a - Used to calculate the neutron dose rate off of the tank side.

Tank Z-361, settling tank, riser uncovered

c neutron dose at side of tank

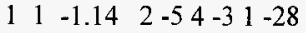
$24-2.258 \quad 2-5-34-17$
$34-2.258-260-54-67$
$4 \quad 4-2.258-93-54-67$

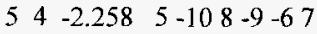

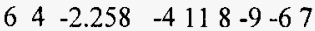
$74-2.258-260-54-126$
$84-2.258-93-54-126$
$94-2.2585-108-9-126$
$10 \quad 4-2.258 \quad 8-911-4 \quad 6-12$

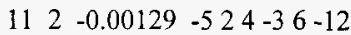
$124 \quad-2.258 \quad 8-10-9111434-13$

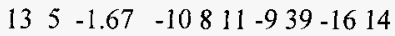
$\begin{array}{llllll}16 & 2 & -0.00129 & -15 & 12 & -17\end{array}$

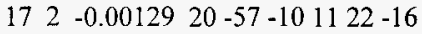
$18 \quad 5 \quad-1.67-219-101122-16$
$195-1.67 \quad 2010-18-21-1622$
$205-1.6720-11 \quad 19-21-1622$
$\begin{array}{lllllllll}21 & 5 & -1.67 & -9 & 8 & -10 & 11 & -7 & 22\end{array}$
$\begin{array}{lllllll}22 & 3 & -7.86 & 15 & -14 & -17 & 12\end{array}$
$\begin{array}{lllllll}23 & 2 & -0.00129 & 14 & -23 & 16 & -17\end{array}$

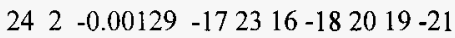

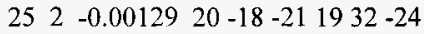
$262-0.0012920-18-21 \quad 1924-25$
$270(((((18)):(-19))):(21-1819)):(-1819-20)):$
$(20-18-211925)):(20-18-2119-22)$
$\begin{array}{lllllll}37 & 1 & -1.14 & 47-5 & 4-3 & -691 & \$ \text { top } 6 " \text { of source }\end{array}$
$\begin{array}{llllllllll}38 & 1 & -1.14 & 47 & -5 & 4 & -3 & -91 & 92 & \$ \text { next } 12 " \text { of source }\end{array}$
$\begin{array}{llllllllll}39 & 1 & -1.14 & 47 & -5 & 4 & -3 & -92 & 28\end{array}$

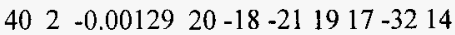
$\begin{array}{llllll}41 & 2 & -0.00129 & 17 & -32 & -14\end{array}$
$42 \quad 4 \quad-2.258 \quad 8 \quad-10-9111412-33$
$\begin{array}{llllllllll}43 & 4 & -2.258 & 8 & -10 & -9 & 11 & 14 & 33 & -34\end{array}$

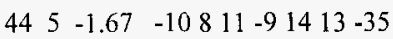

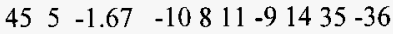

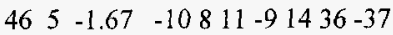

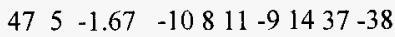




\section{HNF-3361, Rev.0}

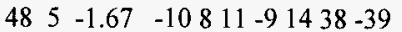

$\begin{array}{llllllll}51 & 1 & -1.14 & 2 & -45 & 4 & -5 & -626\end{array}$

$\begin{array}{lllllllll}52 & 1 & -1.14 & 2 & -45 & 4 & -5 & -26 & 27\end{array}$

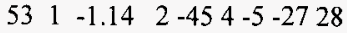

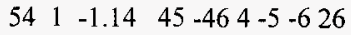

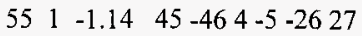

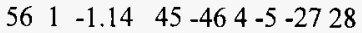

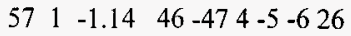

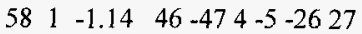

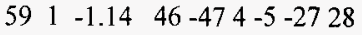

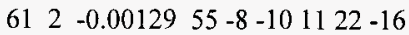

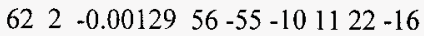

$\begin{array}{lllllllll}63 & 2 & -0.00129 & 57 & -56 & -10 & 11 & 22 & -16\end{array}$

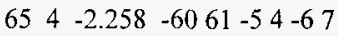

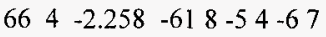

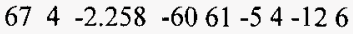

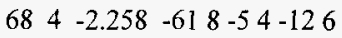

$\begin{array}{cccccc}1 & \mathrm{p}-3.8460000 \mathrm{e}-02 & 0.0000000 & 0.9615400 & 0.0000000 \\ 2 & \mathrm{px} & -396.24000 & & & \\ 3 & \mathrm{px} & 396.24000 & & & \\ 4 & \mathrm{py} & -198.12000 & & & \\ 5 & \mathrm{py} & 198.12000 & & & \\ 6 & \mathrm{pz} & 243.84000 & & & \\ 7 & \mathrm{p}-3.8460000 \mathrm{e}-02 & 0.0000000 & 0.9615400 & -30.480000 \\ 8 & \mathrm{px} & -426.72000 & & & \\ 9 & \mathrm{px} & 426.74000 & & & \\ 10 & \mathrm{py} & 228.60000 & & & \\ 11 & \mathrm{py} & -228.60000 & & & \\ 12 & \mathrm{pz} & 533.40000 & & & \\ 13 & \mathrm{pz} & 563.88000 & & & \\ 14 & \mathrm{c} / \mathrm{z} & 59.436000 & 0.0000000 & 8.4137500 & \\ 15 & \mathrm{c} / \mathrm{z} & 59.436000 & 0.0000000 & 7.7025500 & \\ 16 & \mathrm{pz} & 624.84000 & & & \\ 17 & \mathrm{pz} & 701.04000 & & & \\ 18 & \mathrm{py} & 1752.6000 & & & \\ 19 & \mathrm{py} & -1752.6000 & & & \\ 20 & \mathrm{px} & -1950.7200 & & & \\ 21 & \mathrm{px} & 1950.7200 & & & \\ 22 & \mathrm{pz} & -1600.0000 & & & \\ 23 & \mathrm{c} / \mathrm{z} & 59.436000 & 0.0000000 & 84.613750 & \end{array}$


HNF-3361, Rev.0

\begin{tabular}{|c|c|c|c|c|}
\hline 24 & $\mathrm{pz}$ & \multicolumn{3}{|c|}{777.24000} \\
\hline 25 & $\mathrm{pz}$ & \multicolumn{3}{|c|}{2301.2400} \\
\hline 26 & $\mathrm{pz}$ & \multicolumn{3}{|c|}{182.88000} \\
\hline 27 & $\mathrm{pz}$ & \multicolumn{3}{|c|}{121.92000} \\
\hline 28 & $\mathrm{pz}$ & \multicolumn{3}{|c|}{60.960000} \\
\hline 32 & $\mathrm{pz}$ & \multicolumn{3}{|c|}{703.550} \\
\hline 33 & $\mathrm{pz}$ & \multicolumn{3}{|l|}{543.56} \\
\hline 34 & $\mathrm{pz}$ & \multicolumn{3}{|l|}{553.72} \\
\hline 35 & $\mathrm{pz}$ & \multicolumn{3}{|l|}{574.04} \\
\hline 36 & $\mathrm{pz}$ & \multicolumn{3}{|l|}{584.20} \\
\hline 37 & $\mathrm{pz}$ & \multicolumn{3}{|l|}{594.36} \\
\hline 38 & $\mathrm{pz}$ & \multicolumn{3}{|l|}{604.52} \\
\hline 39 & $\mathrm{pz}$ & \multicolumn{3}{|l|}{614.68} \\
\hline 40 & $\mathrm{c} / \mathrm{z}$ & 59.436 & 0.000 & 30.00 \\
\hline 41 & $c / z$ & 59.436 & 0.000 & 60.00 \\
\hline 42 & $c / z$ & 59.436 & 0.000 & 120.00 \\
\hline 45 & $\mathrm{px}$ & \multicolumn{3}{|c|}{-365.76} \\
\hline 46 & $\mathrm{px}$ & \multicolumn{3}{|l|}{-335.28} \\
\hline 47 & $\mathrm{px}$ & \multicolumn{3}{|l|}{-304.80} \\
\hline 50 & $\mathrm{pz}$ & \multicolumn{3}{|l|}{650.24} \\
\hline 51 & $\mathrm{pz}$ & \multicolumn{3}{|l|}{675.64} \\
\hline 55 & $\mathrm{px}$ & \multicolumn{3}{|l|}{-526.72} \\
\hline 56 & $\mathrm{px}$ & \multicolumn{3}{|l|}{-626.72} \\
\hline 57 & $\mathrm{px}$ & \multicolumn{3}{|l|}{-726.72} \\
\hline 60 & $\mathrm{px}$ & \multicolumn{3}{|l|}{-406.40} \\
\hline 61 & $\mathrm{px}$ & \multicolumn{3}{|l|}{-416.56} \\
\hline \multicolumn{5}{|c|}{ tally segment surfaces } \\
\hline 70 & py & \multirow{2}{*}{\multicolumn{3}{|c|}{$\begin{array}{l}30.48 \\
91.44\end{array}$}} \\
\hline 71 & py & & & \\
\hline 72 & py & \multicolumn{3}{|l|}{152.40} \\
\hline 73 & py & \multicolumn{3}{|l|}{-30.48} \\
\hline 74 & py & -91.44 & & \\
\hline 75 & py & -152.40 & & \\
\hline 76 & $\mathrm{pz}$ & 304.80 & & \\
\hline 77 & $\mathrm{pz}$ & 365.76 & & \\
\hline $\begin{array}{l}78 \\
79\end{array}$ & pz & 426.72 & & \\
\hline 80 & $\begin{array}{l}\mathrm{pz} \\
\mathrm{pz}\end{array}$ & $\begin{array}{c}487.68 \\
0.0\end{array}$ & & \\
\hline 91 & $\mathrm{pz}$ & 228.60 & $\$ 6 "$ be & low top of source \\
\hline 92 & $\mathrm{pz}$ & 198.12 & $\$ 18^{\prime \prime}$ be & elow top of source \\
\hline
\end{tabular}


HNF-3361, Rev.0

mode $\mathrm{n}$

$\mathrm{ml} 13027.50 \mathrm{c}-0.30420000 .50 \mathrm{c}-0.46026000 .55 \mathrm{c}-0.5622$ \$source $1.14 \mathrm{~g} / \mathrm{cc}$ $11023.50 \mathrm{c}-0.040514000 .50 \mathrm{c}-0.01048016 .50 \mathrm{c}-0.200$

$1001.50 \mathrm{c}-0.0606000 .50 \mathrm{c}-0.087217000 .50 \mathrm{c}-0.0342$

$9019.50 \mathrm{c}-0.0039$

$\mathrm{m} 2 \quad 7014.50 \mathrm{c} 0.790008016 .50 \mathrm{c} 0.21000 \quad \$$ air $0.00129 \mathrm{~g} / \mathrm{cc}$

m3 $26000.55 \mathrm{c}-5.100924000 .50 \mathrm{c}-1.56628000 .50 \mathrm{c}-0.9396 \$$ steel $7.86 \mathrm{~g} / \mathrm{cc}$ $25055.50 \mathrm{c}-0.156614000 .50 \mathrm{c}-0.058715031 .50 \mathrm{c}-0.0035$

$16032.50 \mathrm{c}-0.00236000 .50 \mathfrak{c}-0.0023$

$\mathrm{m} 41001.50 \mathrm{c}-0.00318016 .50 \mathrm{c}-0.440711023 .50 \mathrm{c}-0.0182 \$$ concrete $2.258 \mathrm{~g} / \mathrm{cc}$

$12000.50 \mathrm{c}-0.037613027 .50 \mathrm{c}-0.060714000 .50 \mathrm{c}-0.2157$

$15031.50 \mathrm{c}-0.000916032 .50 \mathrm{c}-0.000936084 .50 \mathrm{c}-0.0066$

$20000.50 c-0.130622000 .50 c-0.004926000 .55 c-0.0788$

m5 $8016.50 \mathrm{c}-0.51114000 .50 \mathrm{c}-0.278220000 .50 \mathrm{c}-0.0717 \$$ dirt $1.67 \mathrm{~g} / \mathrm{cc}$

$26000.55 \mathrm{c}-0.109113027 .50 \mathrm{c}-0.0832612000 .50 \mathrm{c}-0.03142$

$19000.50 \mathrm{c}-0.0115511023 .50 \mathrm{c}-0.0202222000 .50 \mathrm{c}-0.01655$

$25055.50 \mathrm{c}-0.00178115031 .50 \mathrm{c}-0.0024$

imp:n 10102 r 102 r 102 r 40 9r 1 2r 0 8r 18 8r 4 2r $2424 \$ 1,53$

c source

c source fraction of total sludge volume (76569 I total volume)

c $w g t=(3.747 \mathrm{e}+05 \mathrm{p} / \mathrm{kg} \mathrm{pu}) \times 30 \mathrm{~kg} \mathrm{pu}=1.124 \mathrm{e}+07$

sdef wgt $=1.124 \mathrm{e}+07 \mathrm{erg}=\mathrm{d} 1 \mathrm{cel}=\mathrm{d} 2$ pos $000 \mathrm{x}$ fcel $\mathrm{d} 3 \mathrm{y}$ fcel $\mathrm{d} 4 \mathrm{z}$ fcel d 5

scl spontaneous fission source spectrum (pu240)

spl -30.7994 .903$

sc2 source cells

si2 15152535455565758593738391

sp2 1111111115.7511 .551 .7526

sb2 1010105.02 .52 .52 .5 .625 .625205 .3125 .15625

ds3 s 31313132323233333334343435

si31 $-396.24-365.76$

sp31 01

si32 $-365.76-335.28$

sp32 01

si33 $-335.28-304.80$

$\operatorname{sp} 3301$

si34 -304.8 396.24

$\mathrm{sp} 3401$

si35 -396.24 396.24

sp35 01

ds4 s 41414141414141414141414141

si41 - 198.12198 .12 
HNF-3361, Rev.0

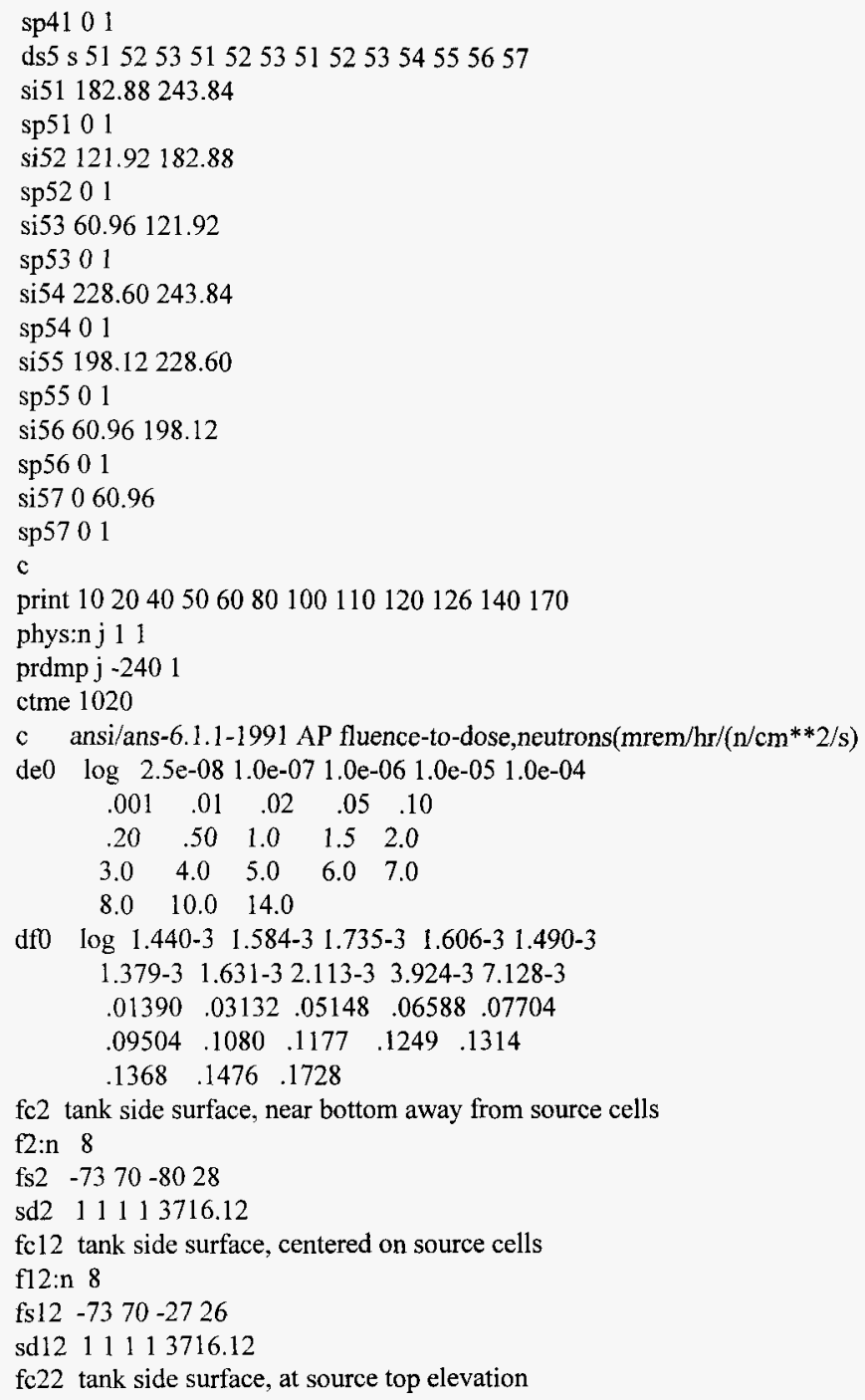

fc2 tank side surface, near bottom away from source cells f2:n 8

fs2 $-7370-8028$

sd2 11113716.12

fc12 tank side surface, centered on source cells

f12:n 8

fs $12-7370-2726$

sd12 11113716.12

fc22 tank side surface, at source top elevation 


$$
\text { HNF-3361, Rev.0 }
$$

f22:n 8

fs $22-7370-676$

sd22 111113716.12

fc32 tank side surface, $7 \mathrm{ft}$ above source top elevation

f32:n 8

fs $32-7370-7879$

$\operatorname{sd} 32 \quad 1111113716.12$

fc42 $1 \mathrm{~m}$ from tank side surface, near bottom away from source cells f $42: n 55$

fs42 $-7370-8028$

sd42 111113716.12

fc52 $1 \mathrm{~m}$ from tank side surface, centered on source cells

f52:n 55

fs52 $-7370-2726$

sd52 111113716.12

fc62 $1 \mathrm{~m}$ from tank side surface, at source top elevation

f62:n 55

fs $62-7370-676$

sd62 111113716.12

fc72 $1 \mathrm{~m}$ from tank side surface, $7 \mathrm{ft}$ above source top elevation

f 72 :n 55

fs $72-7370-7879$

sd72 111113716.12

fc82 $2 \mathrm{~m}$ from tank side surface, near bottom away from source cells f82:n 56

fs $82-7370-8028$

sd82 1111113716.12

fc92 $2 \mathrm{~m}$ from tank side surface, centered on source cells

f92:n 56

fs92 $-7370-2726$

sd92 111113716.12

fc102 $2 \mathrm{~m}$ from tank side surface, at source top elevation

f102:n 56

fs $102-7370-676$

sd102 111113716.12

fc112 $2 \mathrm{~m}$ from tank side surface, $7 \mathrm{ft}$ above source top elevation

f112:n 56

fs112 $-7370-7879$

sd112 111113716.12

fc122 $3 \mathrm{~m}$ from tank side surface, near bottom away from source cells f122:n 57 
HNF-3361, Rev.0

fs $122-7370-8028$

sd122 111113716.12

fc132 $3 \mathrm{~m}$ from tank side surface, centered on source cells

f132:n 57

fs $132-7370-2726$

$\operatorname{sd} 132 \quad 111113716.12$

fc142 $3 \mathrm{~m}$ from tank side surface, at source top elevation

f1 142:n 57

fs $142-7370-676$

sd142 111113716.12

fc152 $3 \mathrm{~m}$ from tank side surface, $7 \mathrm{ft}$ above source top elevation

f1 $152:$ : 57

fs $152-7370-7879$

$\operatorname{sd} 152 \quad 111113716.12$

e 0.10 .30 .61 .32 .33 .045 .17 .112 
HNF-3361, Rev.0

\section{APPENDIX B \\ Correspondence with Lee Carter}

The following MCNP input file was provided as an example by Lee Carter to help improve the statistical uncertainties of the calculated dose rates.

Author: Glcar39@aol.com at $\sim$ EXCHANGE

Date: 8/17/98 7:10 PM

Priority: Normal

BCC: Jay S Lan at $\sim$ HANFORD07A

TO: Jay_s_lan@rl.gov at $\sim$ EXCHANGE

Subject: TANK MCNP FILE ATTACHED Message Contents

task W-151, tank AZ-101, file i21

c bottom of tank

$1 \quad 520.0652-1051-2$

c radial side of tank

$2 \quad 3-7.8000 \quad 101-1022-112$

$353-0.0012((-111102-1032):(111102-22-112)):(11221-22$

)

$4 \quad 3 \quad-7.8000 \quad 103-1042-111$

$5 \quad 52 \quad 0.0652 \quad 2-105104-111$

c. top of tank

$\begin{array}{lllllll}6 & 3 & -7.8000 & 112 & 20 & -21 & -22\end{array}$

$\begin{array}{llllll}7 & 3 & -7.8000 & 22 & -23525456586062111\end{array}$

$8 \quad 520.0652 \quad 23-105((-25107):(-24-107)) 525456586062111$

c dummy concrete cells within dome for risers

$\begin{array}{lllllll}11 & 52 & 0.0652 & 111 & 22 & -24 & -52\end{array}$

$\begin{array}{llllllll}12 & 52 & 0.0652 & 111 & -54 & 22 & -24\end{array}$

$\begin{array}{lllllll}13 & 52 & 0.0652 & 111 & -56 & 22 & -24\end{array}$

$\begin{array}{llllllll}14 & 52 & 0.0652 & 111 & -58 & 22 & -24\end{array}$

$\begin{array}{llllllll}16 & 52 & 0.0652 & 111 & -62 & 22 & -24\end{array}$

c soil beyond tank

$31 \quad 1-1.67 \quad 111-113-10660(152:-115)(25:-107)(24: 107)$

$32 \quad 1-1.67 \quad 1105-106((111-25):-111)$

c inside of tank

$41 \quad 61-1.70 \quad 2-121-101$ \$ sludge

$42 \quad 62-1.21 \quad 121-122-101$ \$ supernantant beyond source region used $(-177: 854)(133:-141)$

c source region used

$43 \quad 62-1.21 \quad-131 \quad 143-122$ 
HNF-3361, Rev.0

854

$$
\begin{aligned}
& \begin{array}{lllllll}
44 & 62 & -1.21 & -132 & 131 & 143 & -122
\end{array} \\
& \begin{array}{llllllll}
45 & 62 & -1.21 & -133 & 132 & 143 & -122
\end{array} \\
& \begin{array}{llllll}
46 & 62 & -1.21 & -131 & 142 & -143
\end{array} \\
& 854 \\
& \begin{array}{lllllll}
47 & 62 & -1.21 & -132 & 131 & 142 & -143
\end{array} \\
& \begin{array}{lllllll}
48 & 62 & -1.21 & -133 & 132 & 142 & -143
\end{array} \\
& \begin{array}{lllllll}
49 & 62 & -1.21 & -131 & 141 & -142
\end{array} \\
& 854 \\
& \begin{array}{llllllll}
50 & 62 & -1.21 & -132 & 131 & 141 & -142
\end{array} \\
& \begin{array}{llllllll}
51 & 62 & -1.21 & -133 & 132 & 141 & -142
\end{array} \\
& 52 \quad 53-.0012 \quad 122-101((112-20-22):-112) \quad \$ \text { above liquid } \\
& 854(-173: 60) \\
& \text { c steam coil riser }
\end{aligned}
$$

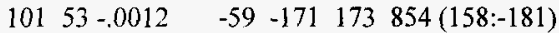

$$
\begin{aligned}
& ((156:-189)(156: 164: 189: 187)(164:-187: 188) \\
& (164: 166:-189:-188)(166: 189:-190))
\end{aligned}
$$

$\begin{array}{lllllll}102 & 3 & -7.8 & 59 & -60 & -171 & 173\end{array}$

$$
\begin{aligned}
& \text { c steam coil flange above riser }
\end{aligned}
$$

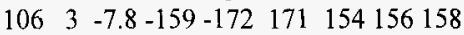

$$
\begin{aligned}
& \text { c steam coil pipes } \\
& \text { c up to bottom of flange at top of riser } \\
& 11153-.0012 \quad-153178-171 \quad \$ \text { \#5 - } x \\
& 112 \quad 3-7.8-854177-171(153:-178) \\
& 11353-.0012 \quad 190-171 \quad \$ 4 " \text { cnds } \\
& ((-155189):(-155-163-189-187):(-163187-188) \text { : } \\
& (-163-165189188):(-165-189))
\end{aligned}
$$

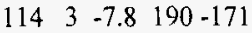$$
((-156189):(-156-164-189-187):(-164187-188) \text { : }
$$$$
(-164-166189188):(-166-189)) \quad \# 113
$$

$115 \quad 53-.0012 \quad-157181-171 \$$ \$ $3-x$

$\begin{array}{lllllll}116 & 3 & -7.8 & -158 & 181 & -171 & 157\end{array}$

c $\quad$ 1st imp zone above top of riser

$11753-.0012 \quad-153171-183 \quad \$$ \#5 -x

$\begin{array}{llllllll}118 & 3 & -7.8 & -154 & 171 & -183 & 153\end{array}$

$\begin{array}{llllll}119 & 53 & -.0012 & -155 & 171 & -183\end{array} \$ \# 5+x$

$\begin{array}{llllllll}120 & 3 & -7.8 & -156 & 171 & -183 & 155\end{array}$

$12153-.0012 \quad-157 \quad 171-183 \$$ \$ $\quad$-x

$\begin{array}{lllllll}122 & 3 & -7.8 & -158 & 171 & -183 & 157\end{array}$

c 2nd imp zone above top of riser

$123 \quad 53-.0012-153 \quad 183-184 \$ \# 5-x$ 
HNF-3361, Rev.0

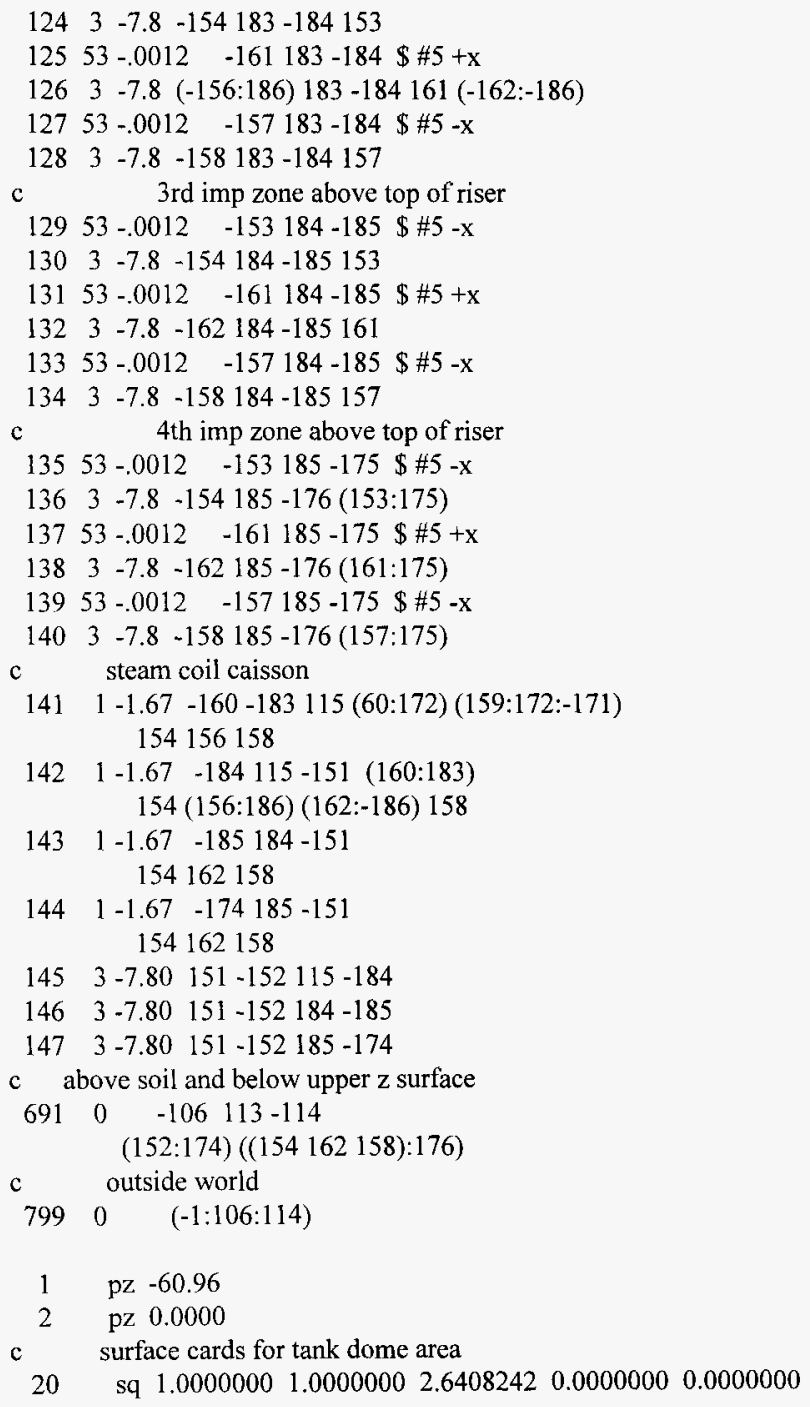


HNF-3361, Rev.0

$0.0000000-1306449.0000000 \quad 0.0000000 \quad 0.0000000 \quad 1072.6229500$

21 sq $1.00000000 \quad 1.00000000 \quad 2.66667502 \quad 0.00000000 \quad 0.00000000$ $\begin{array}{lllll}0.00000000 & -1308627.32200000 & 0.00000000 & 0.00000000 & 1072.62295000\end{array}$

22 sq $1.00000000 \quad 1.00000000 \quad 7.14417474 \quad 0.00000000 \quad 0.00000000$ $0.00000000-1486448.64000000 \quad 0.00000000 \quad 0.00000000968 .95920000$

23 sq $1.0000000000 \quad 1.00000000007 .1255520600 \quad 0.0000000000$ $0.0000000000 \quad 0.0000000000-1488772.12299999990 .0000000000$ 0.0000000000968 .9592000000

24 sq $1.00000000 \quad 1.00000000 \quad 6.47143574 \quad 0.00000000 \quad 0.00000000$ $0.00000000-1580803.29000000 \quad 0.00000000 \quad 0.00000000968 .95920000$

25 sq $\quad \begin{array}{llllll}1.00000000 & 1.00000000 & 2.20454312 & 0.00000000 & 0.00000000\end{array}$ $\begin{array}{lllll}0.00000000 & -2316125.44000000 & 0.00000000 & 0.00000000 & 500.00000000\end{array}$

c riser surfaces

$51 \quad 1 \mathrm{cz} 53.34000000$

$52 \quad 1 \mathrm{cz} 54.61000000$

$53 \quad 2$ cz 53.34000000

$54 \quad 2 \mathrm{cz} 54.61000000$

$553 \mathrm{cz} 53.34000000$

$56 \quad 3 \mathrm{cz} 54.61000000$

$57 \quad 4 \mathrm{cz} 53.34000000$

$58 \quad 4 \mathrm{cz} 54.61000000$

$59 \quad 5 \mathrm{cz} 53.34000000$

$60 \quad 5 \mathrm{cz} 54.61000000$

$61 \quad 6 \mathrm{cz} 53.34000000$

$\begin{array}{lll}62 & 6 \mathrm{cz} & 54.61000000\end{array}$

c radial surfaces for tank

101 cz 1143.00000000

102 cz 1143.95250000

103 cz 1219.20000000

104 cz 1220.15250000

105 cz 1264.92000000

106 cz 1325.00000000

$107 \mathrm{cz} 1095.85125000$

c $\quad z$-surfaces for outside of tank

111 pz 968.95900000

112 pz 1072.62300000

113 pz 1696.98 \$ top of soil

114 pz 1820. \$top of model

$115 \mathrm{pz} 1658.88$ base of caisson

c $\quad$-surfaces for inside of tank

121 pz 50.8 \$ top of sludge 
HNF-3361, Rev.0

$122 \mathrm{pz} 914.4$ \$ top of supernatant

c radial surfaces for source in supernatant

$1315 \mathrm{cz} 91.44$

$1325 \mathrm{cz} 182.88$

$1335 \mathrm{cz} 365.76$

c axial surfaces for source in supernatant

141 pz 822.96

142 pz 853.44

$143 \mathrm{pz} 883.92$

$149 \mathrm{pz} 1727.46 \$$ for tally, radial edge of caisson

c surfaces for steam coil

$1515 \mathrm{cz} 91.12$ \$ inside of earth filled caisson

$1525 \mathrm{cz} 91.44$ \$ outside of earth filled caisson

$1535 \mathrm{c} / \mathrm{z} 45.720 .3 .8964$ \$ inside of pipe \#5

$1545 \mathrm{c} / \mathrm{z} 45.720 .4 .445$ \$ outside of pipe \#5

$155 \quad 5 \mathrm{c} / \mathrm{z}-45.720 .3 .8964$ \$ inside of pipe \#5

$1565 \mathrm{c} / \mathrm{z}-45.720 .4 .445$ \$ outside of pipe \#5

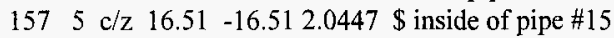

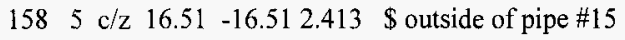

$\begin{array}{lll}159 \quad 5 \mathrm{cz} & 63.50 \quad \text { \$ flange at top of riser }\end{array}$

$1605 \mathrm{cz} 72.0 \quad$ \$ importance surface for caisson

$1615 \mathrm{c} / \mathrm{z}-45.720 .2 .62509$ \$ inside of $2 "$ cnds

$1625 \mathrm{c} / \mathrm{z}-45.720 .3 .01625$ \$ outside of $2 "$ cnds

$1639 \mathrm{cx} 3.8964 \$$ inside 4" cnds, jog below flange

$1649 \mathrm{cx} 4.445$ \$ outside 4" cnds, jog below flange

$1655 \mathrm{c} / \mathrm{z} \quad 0 .-45.723 .8964$ \$ inside $4 " \mathrm{cnds}, \mathrm{b}$ jog b flange

$1665 \mathrm{c} / \mathrm{z} \quad 0 .-45.724 .445$ \$ outside 4 " cnds, b jog b flange

$171 \mathrm{pz} \quad 1712.22 \$$ top of coil riser, bottom of flange

$172 \mathrm{pz} \quad 1716.03 \mathrm{~S}$ top of flange

$173 \mathrm{pz} 1330.0$ \$ bottom of riser ???

$174 \mathrm{pz} \quad 1780.80$ \$ top of caisson

$175 \mathrm{pz} \quad 1804.93$ \$ bottom edge of top of \#5 pipe

$176 \mathrm{pz} 1805.48$ \$ top of \#5 pipe

177 pz 792.48 \$ bottom of \#5 pipe

$178 \mathrm{pz} 793.0286$ \$ bottom of \$5 pipe 153,154

179 pz 1681.74 \$ bottom of \#5 pipe 155,156

$180 \mathrm{pz} \quad 1682.2886$ \$ bottom of \#5 pipe 155,156

$181 \mathrm{pz} 1437.9$ \$ bottom of \#15 pipe 157,158

c $\quad 182 \mathrm{pz} \quad 152.7683$ \$ bottom of \#15 pipe 157,158

$183 \mathrm{pz} \quad 1732.2$ \$ importance surface for caisson

$184 \mathrm{pz} \quad 1748.4$ \$ importance surface for caisson 


\section{HNF-3361, Rev.0}

$185 \mathrm{pz} \quad 1764.6$ \$ importance surface for caisson

186 pz $1732.7486 \$$ above reduction in $2 " \mathrm{cnds}$

$1879 \mathrm{px} 0$. \$ plane in cnds, 1 st jog below flange

1889 px 64.65784407 \$ plane in cnds, 2nd jog below flange

189 pz 1685.55 \$ jog of 4" cnds below flange

$190 \mathrm{pz} 1655.07$ \$ lower fake end of 4 " ends below flange

$3545 \mathrm{c} / \mathrm{z} 45.720 .6 .985 \$ 1$ " beyond $4 "$ steam for tally

$4545 \mathrm{c} / \mathrm{z} 45.720 .9 .525 \$ 2$ " beyond 4 " steam for tally

$3625 \mathrm{c} / \mathrm{z}-45.720 .5 .55625 \$ \mathrm{I}$ " beyond $2 " \mathrm{cnds}$ for tally

$4625 \mathrm{c} / \mathrm{z}-45.720 .8 .09625 \$ 1$ " beyond $2 " \mathrm{cnds}$ for tally

$3585 \mathrm{c} / \mathrm{z} \quad 16.51-16.514 .953 \$ 1$ " beyond $1.5^{\prime \prime}$ pipe for tally

$4585 \mathrm{c} / \mathrm{z} \quad 16.51-16.517 .493 \$ 2^{\prime \prime}$ beyond $1.5^{\prime \prime}$ pipe for tally

$374 \quad \mathrm{pz} \quad 1785.8 \quad \$ 2^{\prime \prime}$ above caisson for tally

$375 \mathrm{pz} 1801.04 \$ 8$ " above caisson for tally

$8545 \mathrm{c} / \mathrm{z} 45.720 .4 .445001$ \$ outside of pipe \#5

mode $\mathrm{p}$

imp:p $\quad 003 \mathrm{r} .257 \mathrm{r} \quad \$ 16$

10001.25 .0625 .25 .0625 .015625 .0625 .015625

.0003906251 . $\$ 52$

11415 r 85 r 325 r 1285 r 5125 r $\$ 140$

8321285128321285120 .

phys:p $\mathrm{j} 1$

ml 8000.01p -.511 14000.01p -.2782 20000.01p -.0717 \$ hwvp dirt $26000.01 \mathrm{p}-.109113000 .01 \mathrm{p}-.0832612000 .01 \mathrm{p}-.03142$ \$ hwvp dirt $19000.01 \mathrm{p}-.0115511000 .01 \mathrm{p}-.0202222000 .01 \mathrm{p}-.01655 \$$ hwvp dirt $25000.01 \mathrm{p}-.00178115000 .01 \mathrm{p}-.0024 \quad \$$ hwvp dirt

m3 $26000.01 \mathrm{p}-1$.

m52 $1000.01 \mathrm{p} 0.0642 \quad 8000.01 \mathrm{p} 0.5916 \quad 14000.01 \mathrm{p} 0.2405$

$20000.01 \mathrm{p} 0.073826000 .01 \mathrm{p} 0.0299 \quad \$$ ordinary H concrete

m53 7000.01p -0.765 8000.01p-0.235 \$ air

c slude (with $60 \%$ supernate), EDT 155422, page 11, 1-initial

m61 $\quad 1000.01 \mathrm{p} 25322 \quad 6000.01 \mathrm{p} 413.6 \quad 7000.01 \mathrm{p} 11284$

$8000.01 \mathrm{p} 299001 \quad 9000.01 \mathrm{p} 298.5 \quad 11000.01 \mathrm{p} 37914$

$13000.01 \mathrm{p} 2046 \quad 15000.01 \mathrm{p} 86.0 \quad 16000.01 \mathrm{p} 1449$

$19000.01 \mathrm{p} 786.0 \quad 24000.01 \mathrm{p} 6266 \quad 26000.01 \mathrm{p} 69518$

$40000.01 \mathrm{p} 23888 \quad 14000.01 \mathrm{p} 4955 \quad 48000.01 \mathrm{p} 7754$

$38000.01 \mathrm{p} 55.1 \quad 44000.01 \mathrm{p} 0.19 \quad 55000.01 \mathrm{p} 3.24$

$58000.01 \mathrm{p} 0.24 \quad 92000.01 \mathrm{p} 9009 \quad 94000.01 \mathrm{p} 38.2$

95000.01 p 192.3

c supernate, EDT 155422, page 11, 1-initial 
HNF-3361, Rev.0

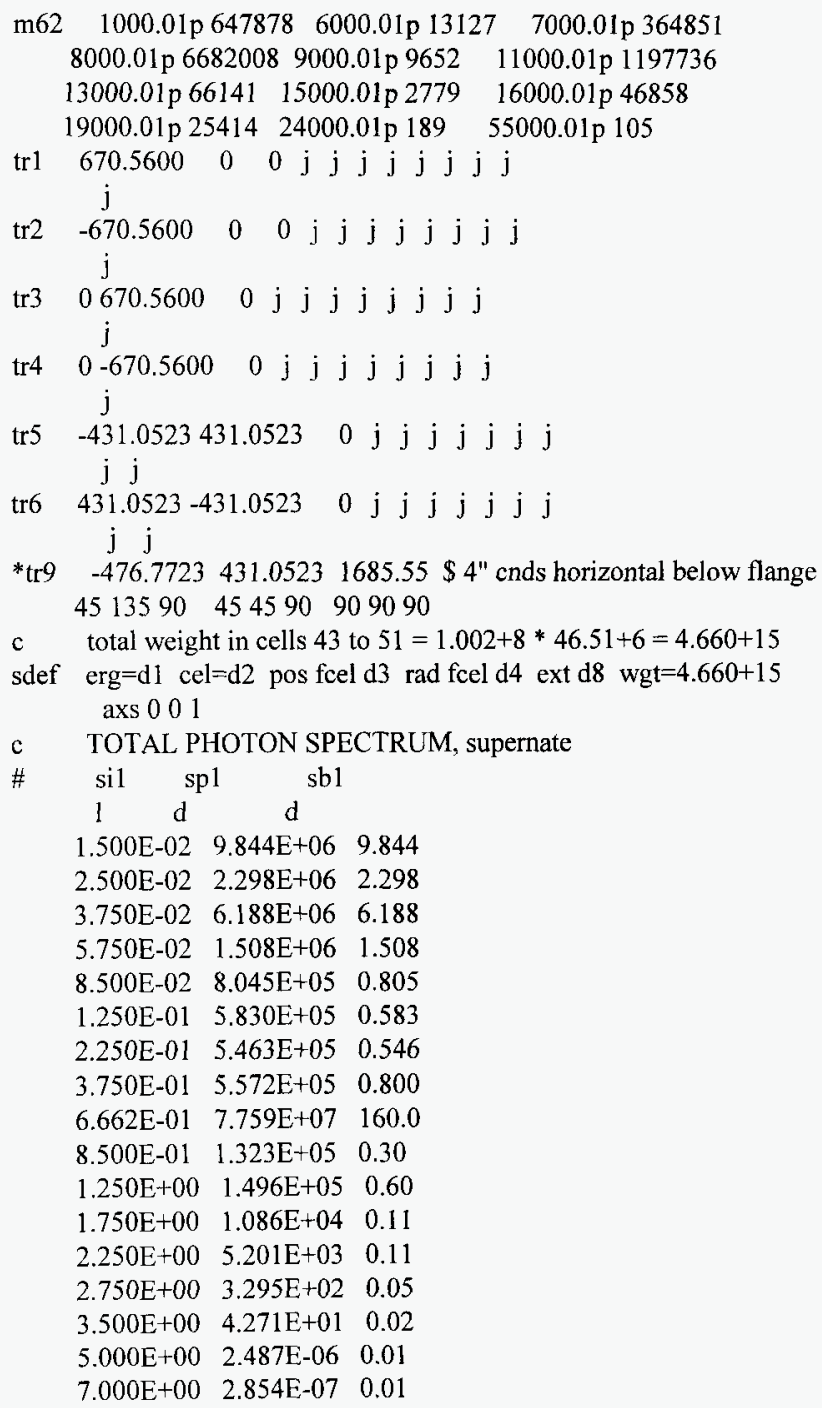


HNF-3361, Rev.0

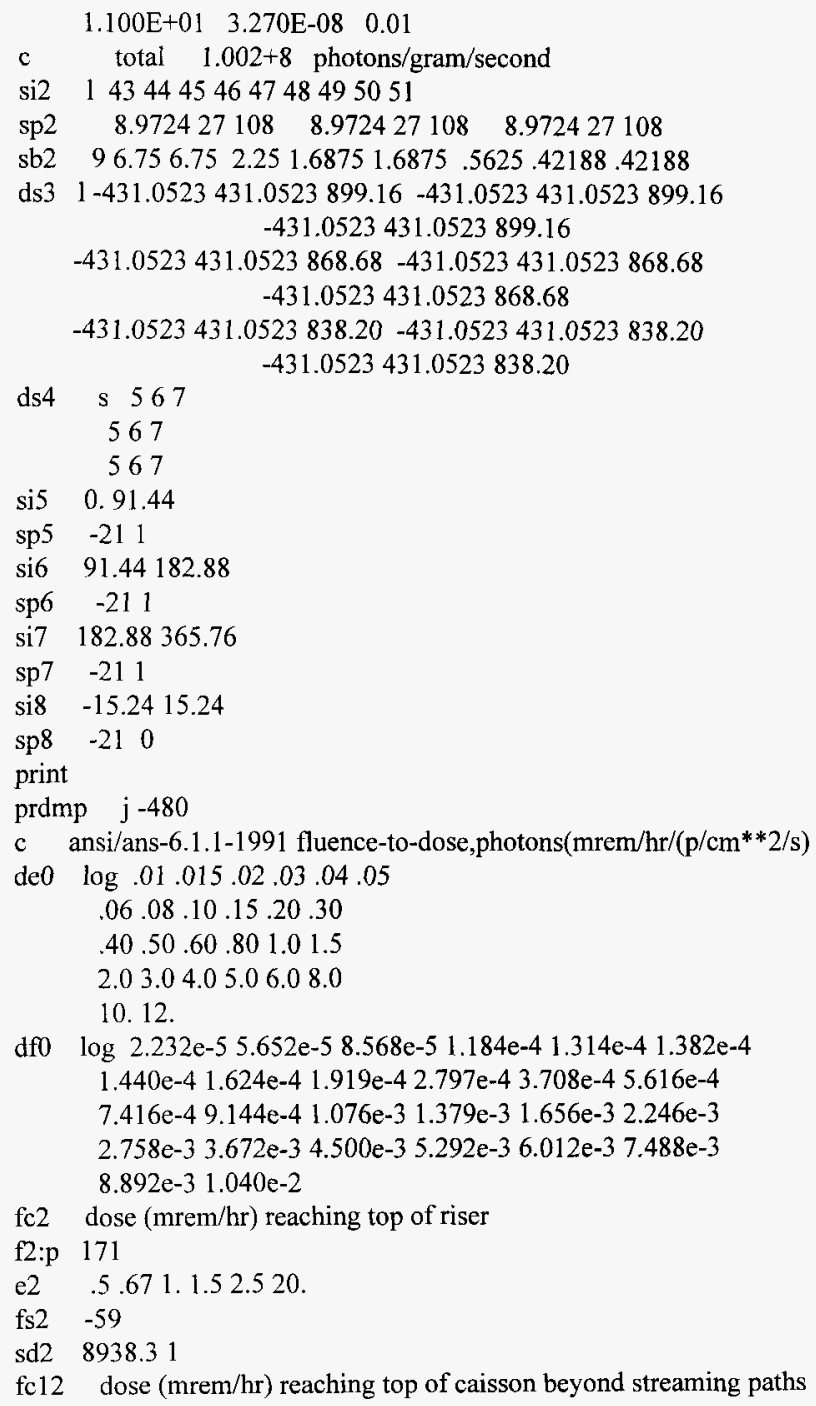


HNF-3361, Rev.0

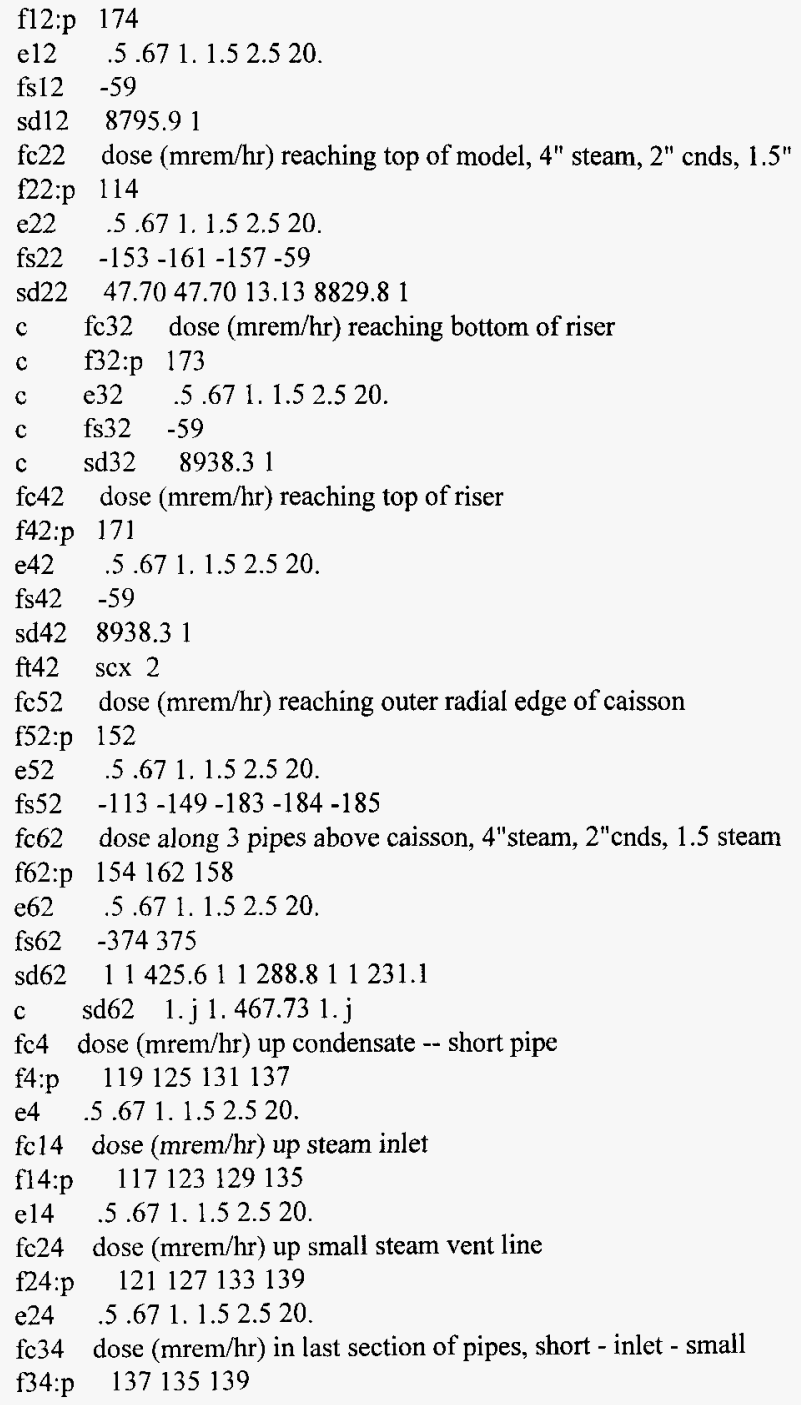




\section{HNF-3361, Rev.0}

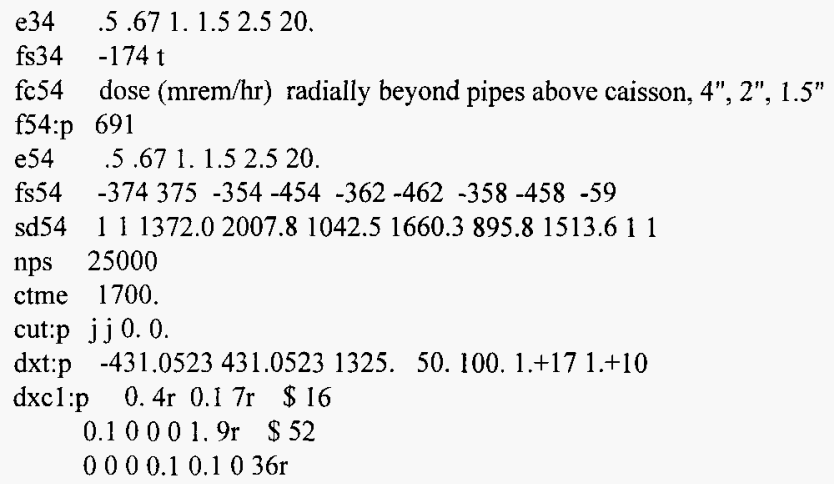




\section{HNF-3361, Rev.0}

Following are communications with Lee Carter

Author: Glcar39@aol.com at $~ E X C H A N G E$

Date: 9/4/98 11:04 AM

Priority: Normal

BCC: Robert F Richard at $\sim$ HANFORD07A

TO: Jay_s_lan@rl.gov at $\sim$ EXCHANGE

TO: Robert_F_Richard@rl.gov at EXCHANGE

Subject: REVIEW OF PHOTON PORTION OF TANK Z-361

Message Contents

Jay and Bob,

I have reviewed the photon portion of the summary and the input files. The attached Word Perfect 6 file contains my comments. I thought I would give this to you now since there is some other work I must get to. Will try to look at the neutron portion in a few days.

Note that I have made some revised calculations, where some modifications were made in the input files to attempt to improve the efficiency and do some other fine tuning. Will send these two input files to you separately.

Best regards,

Lee Carter

TECHNICAL REVIEW OF DOSE RATE CALCULATIONS FOR TANK Z-361

September 14, 1998

Review by:

Dr. Lee L. Carter

Carter Monte Carlo Analysis

6636 Hogan Drive N.

Keizer, OR 97303

Phone (503) 304-2966

Fax (503) 304-2967

E-mail Glcar39@aol.com

Following is a summary of the review, where there are separate discussions of the photon 


\section{HNF-3361, Rev.0}

calculations and of the neutron calculations. The discussion of the photon calculations was made available previously. No errors were identified in the neutron calculations, but a number of suggestions are given on how to make these types of Monte Carlo calculations more efficient.

\section{PHOTON CALCULATIONS}

The summary and MCNP input files po361 and ps 361 have been reviewed. These Monte Carlo calculations are quite difficult and the input files represent a sophisticated treatment. However, there are some concerns/recommendations that, if addressed, may change the calculated dose rates.

The concerns/recommendations for the po361 MCNP input file, used to determine dose rates above and around the open riser, are:

1. The energy biasing probability on the sblcard is available to improve the efficiency of the calculation. This is accomplished by computing each entry on this card as the product of the corresponding source strength entry on the splcard and an estimate of the importance at that source energy. In shielding dose-rate problems the importance essentially always increases with photon energy. However, the sbl entries for the po361 file must have been determined some other way, and some of the entries actually correspond to a decrease instead of an increase in the importance function with photon energy. This likely doesn't make much difference for the dose rate above the riser since the large low energy photon portion of the source tends to dominate at the top of the riser, but it may lead to poor statical uncertainties and a tendency to underestimate the dose rates radially beyond the riser.

2. The three concrete cells, $(42,43$, and 12$)$, should probably have entries of zero rather than one on the dxc:p card because this tends to force scattering back down to the sphere and makes the problem less efficient.

3. Inclusion of the input card cut:p j j 00

is recommended to eliminate the random walk weight check leaving dxtran sphere.

4. The po361 input file was modified to incorporate the above three comments, with the most important being the first comment, and a 30 minute calculation was made on a $200 \mathrm{MH}$ Pentium. The resulting tallies appear to have reduced uncertainties from this short calculation and the results give improved contributions from the higher energies, and actually may change the answers significantly out the side. It is suggested that the changes in this input file be examined, any other appropriate changes incorporated, and a full length calculation be made with the modified input file. 
HNF-3361, Rev.0

The concerns/recommendations for the ps 36 a MCNP input file, used to determine dose rates beyond the side of the tank, are:

1. The same comment is pertinent here as comment $\# 1$ for the po361 input file regarding the energy bias sb 1 card. In this problem, the energy biasing is more important because of attenuation through the concrete.

2. The top of the source is neglected for distances greater than three feet from the wall. However, this portion of the source likely makes a significant contribution for the dose rates above the elevation of the top of the source.

3. The importances were set to zero for the ceiling and walls (except for the tally wall) above source. This scattering contribution should either be included in the calculation or a justification for neglecting it should be given. It probably is small for photons.

4. The ps36a input file was modified to incorporate the above three comments, some additional fine tuning of the importances was made, and a 30 minute calculation was made on a $200 \mathrm{MH}$ Pentium. The resulting dose rates give better statistics in general, especially for the higher energies. The dose rate at the 6 to 8 foot elevation on the outer wall surface still has large uncertainties in this short calculation, but the results indicate that the dose rate there may be two orders of magnitude higher than given in the summary. It is suggested that the changes in this input file be examined, any other appropriate changes incorporated, and a full length calculation be made with the modified input file.

The following are some additional questions and editing comments that you may want to consider:

1. Many of the results shown in the tables are with large statistical uncertainties, and sometimes dose rates increase (or decrease) when one would physically expect the opposite. It should be stated in the text, or tables, or both that dose rates with statistical uncertainties greater than $20 \%$ are order of magnitude estimates only, and the actual values may be off by factors of two or more.

2. In Table 2.1, rather than showing the dose rate as 0.0 above the riser with one inch of steel, perhaps it should either be quantified or a footnote included stating that it is "very small". Also consider including a clarifying footnote that the "dose rate outside radius of riser" is the average from $\mathrm{r}=8.41 \mathrm{~cm}$ to $\mathrm{r}=84.61 \mathrm{~cm}$.

3. I assume you have looked at the source distribution with the Visual Editor? I have not done 


\section{HNF-3361, Rev.0}

that, but its certainly worthwhile to do.

4. The sludge material, $\mathrm{ml}$, has an interesting composition. The number of oxygen atoms per hydrogen atom is only about 0.84 , while I thought a factor of two or more was typical. Also, the dirt is completely dry with no hydrogen -- is this to be conservative for the neutrons?

5. I did not do any review of the source. Apparently, there isn't any radioactive materials in the tank except plutonium, no fission products?

\section{NEUTRON CALCULATIONS}

The summary and MCNP input files no 361 and ns 361 have been reviewed. No specific errors were found in the review. There are a few comments on each of the input files that you may want to consider, where some of these comments have to do with making the calculations more efficient; i.e., obtaining reliable answers with less computer time.

The comments on the no361 MCNP input file, used to determine dose rates above and around the open riser, are:

1. Its my recommendation to typically use the "cut:n $\mathrm{j} \mathrm{j} 00$ " card and have analog capture for neutrons. The manual often recommends non-analog capture or occasional use of the "phys: $\mathrm{j} 1$ 1 " card, but I don't agree with those recommendations.

2. Its not clear that one is sampling the important part of the source sufficiently or that scatting from the source to the riser vicinity is frequent enough in the random walks in this brute force approach. However, the statistical uncertainties obtained in Table 2.3 do indicate that the problem was ran sufficiently long to obtain reliable information. The following could be done in this type of problem to improve the efficiency, but $2 \mathrm{c}$ of the following is not straightforward -however, even the implementation of $2 a$ and $2 b$ could substantially improve the efficiency:

2a. Create an additional radial zone in the concrete and soil of the roof, where this radial zone extends from the riser out a little beyond where the radial side tally is made. Split the concrete and soil in this radial zone into a number of cells to increase inportances with distance up though these cells. The lowest concrete of the roof in this radial zone would probably have an importance a factor of perhaps 8 (or even 64) greater than the source. The intent is to focus the calculation on the riser vicinity and also with distance up through the concrete and soil.

2b. Create an additional radial zone for each axial cell of the source (or at least for the top cell or two), where this radial zone is centered about the axial center of the riser and its radius is somewhat greater than the line-of-sight up the riser. Then include this in the si2, 
$\mathrm{sp} 2$, and $\mathrm{sb} 2$ source description, where sb2 would be set to sample this radial zone more frequently [usually set each sb2 entry to the corresponding sp2 entry multiplied by a guess importance]. This is to increase sampling the source in the line-of-sight region of the source. For consistency, should also set cell importances within the source region proportional to the importance used to compute the corresponding sb2 entry.

2c. Consider using a DXTRAN sphere (like was done for the photon) with center at the bottom of the riser. Then put zero probabilities on the dxc:n card for all of the roof and above, and decreasing probabilities for distances down into the source - these probabilities could be uniformly decreased if neutrons penetrating up through the concrete and soil are important relative to those up through the riser so one doesn't spend all the time on DXTRAN particles. Its my understanding that the default weight cutoffs of zero on the dxt card do not play roulette with the neutrons upon leaving the DXTRAN sphere, which is good. You don't want to kill these particles with roulette, and can usually tell if they are being terminated from the summary table.

Note: I set up a problem modifying file no361 as described in $2 \mathrm{a}, 2 \mathrm{~b}$, and $2 \mathrm{c}$ above, and ran it for five hours on my slower Pentium $150 \mathrm{MH}$. The resulting dose rates agreed well with those in Table 2.3 of the report. The uncertainties were considerably improved for the tallies at $76.20 \mathrm{~cm}$ from the side of the riser - varying from $8.9 \%$ to $12.8 \%$ for this shorter run. However, the uncertainty of $38 \%$ in the dose rate above the riser and within the riser radius is still poor. Another short 10 minute calculation was made with only the source out to a radius of $56 \mathrm{~cm}$ and for the top 3 feet of the source region to obtain the direct streaming dose rate 30 inches above the top of the riser using a point detector. The result was a dose rate of $5.57 \times 10^{-5}(4.4 \%$ uncertainty) $\mathrm{mrem} / \mathrm{hr}$, where this provides a useful confirmation with a good statistical uncertainty that the direct streaming contribution is small. This result indicates that more than half of the dose rate above the riser is from bulk shield rather than streaming up the riser. These types of problems, where part of the contribution is from bulk shield and part from streaming, tend to be difficult. A further improvement might be obtained by creating more radial cells near the riser in the concrete and soil of the roof and increasing importances near the riser and with increasing elevation to emphasize that vicinity in the calculation.

The comments on the ns361 MCNP input file, used to determine dose rates out the side of the $\underline{\text { tank, are: }}$

1. The source part of the geometry was apparently set up to improve the efficiency with an sb2 card, but an sb2 card wasn't included. Could use an sb2 emphasizing the source near the wall and near the top [high guess importance near the wall and near the top as multiplier to sp2], along with a cell importance distribution consistent with the sb2, and also increase importances 
HNF-3361, Rev.0

at entrance to the dose tally wall by at least a factor of 8 to focus more of the calculation on the wall.

2. Importances are zero in the walls (except for tally wall), even above the source elevation, so wall scattering is being neglected. Should probably either include the walls above the source or else quantify that scattering off them is negligible.

3. Importances are decreased by a factor of two at entrance to the air beyond the wall. That will increase the statistical uncertainties for the tally planes beyond the wall.

The following are some additional questions and editing comments that you may want to consider:

1. Apparently Table 2.3 of the report is mislabeled and should be neutron dose rates rather than gamma.

2. Shouldn't the location in Table 2.4 (Table 2.2 also for the gammas) be " 0 to $2 \mathrm{ft}$ above top" instead of " 1 to $2 \mathrm{ft}$ above top" since that's apparently where the tallies were made?

Subject: Photon Dose rates

Author: Robert F Richard at $\sim$ HANFORD07A

Date: $9 / 9 / 98$ 9:26 AM

Lee,

Thanks for the revised input files. I ran them overnight on the UNIX machines and got much improved uncertainties. For total dose rates over all energies most uncertainties are in the range of a few percent, some 1 to 2 percent or less, and one around 10 percent. Some specific energy groups had uncertainties a bit higher.

The dose rates didn't change much, but those off to the side now decrease with distance as would be expected.

My question is: How does one go about determining the correct biasing? Specifically the relative importance of each energy group.

In my attempt to determine the biasing I did a tally on the inside 


\section{HNF-3361, Rev.0}

surface of the tank top (only air between the source and top). That tally result gave essentially no photons from the higher energy groups. I was obviously off track and would like to know how approach it in the furture.

Have you had a chance to look at the neutron calculations yet?

Thanks,

Bob 
HNF-3361, Rev.0

Author: Glcar39@aol.com at EXCHANGE

Date: 9/9/98 2:37 PM

Priority: Normal

BCC: Robert F Richard at $\sim$ HANFORD07A

TO: Robert_F_Richard@RL.gov at $\sim$ EXCHANGE

Subject: Re: Photon Dose rates

Message Contents

Bob,

One can calculationally determine an energy dependent importance for a given dose rate location by replacing the energy dependence of the source with a monoenergetic, unit-strength source. The dose rate is then proportional to the importance. Then you repeat this with enough unit-strength source calculations at other energies until you can draw a curve through the dose rates (importances); i.e., obtain a curve giving importance as a function of source energy. The problem is that sometimes you are interested in some dose rate locations through a thick shield as well as at other locations with little or no shielding. Then you may need to run a problem for the dose rate locations beyond the thick shield and another problem for dose rate locations with little or no shielding -- calculating an importance function with monoenergetic calculations for each problem type. Sometimes you can use the importance function for the thick shield ok for the thin shield results also -- this may require adjusting the importance function a little at low energies so that you sample the lower energies with the biasing function occasionally. I usually try to adjust my bias probablility distribution so that I'm sampling all energies that I think could possibly be important at least occasionally (at least once every 10,000 source particles, for example).

Its time consuming to calculationally determine an importance function. You can usually assume one, with appropriate modifications to make sure that all possibly important energy groups are sampled at least occasionally. The assumed importance function might be proportional to photon energy, energy squarred, energy cubed, or even stronger with energy, depending upon the shield thickness and the general energy range of the photons. After multiplying the photon probability distribution by this importance function to obtain a bjas function, then I do some more thinking about this bias function. If there is one or more very intense low energy groups that could possibly contribute to the dose and the bias probability is very low, I will increase these so they are sampled occasionally. Similarly, if the very high energy groups could possibly contribute to the dose and the bias probability is very 
low, I will increase these groups so they are sampled occasionally; i.e., really modify the low and/or high energy part of the importance function so all possibly important groups are sampled occasionally in case my guess of the importance function is poor for some tallies.

There can be quite a bit of computation going on in creating the bias probability distribution so its usually a good idea to look at the right hand weight multiplier in the energy table of print table 10 in the outp file as a check on your final bias distribution. This column is inversely proportional to the importance function being used and for photons should typically decrease with increasing photon energy.

Often the neutron importance function is nearly constant as a function of energy so its not even necessary to use a bias distribution.

I'm really pressed for time right now and haven't looked any at the neutron calculations. My plans were to do that this Saturday and next Monday so would probably get any comments I might have to you by Monday afternoon. Will that work in your schedule? I assume that the neutrons are simpler in some aspects than the photons.

Best regards,

Lee Carter

Glcar39@aol.com

Phone (503) 304-2966

Fax (503) 304-2967 
HNF-3361, Rev.0

\section{APPENDIX C: CHECKLIST FOR INDEPENDENT TECHNICAL REVIEW}

DOCUMENT REVIEWED

NUMBER:HNF-3361, Rev. 0

TITLE: Tank Z-361 Dose Rate Calculations

\section{AUTHOR(s): J, S. Lan}

I. Method(s) of Review

(X) Input data checked for accuracy

( ) Independent calculation performed

( ) Hand calculation

( ) Alternate computer code:

( ) Comparison to experiment or previous results

( ) Alternate method (define)

II. Checklist (either check or enter NA if not applied)

(X) Task completely defined

(x) Activity consistent with task specification

(x) Necessary assumptions explicitly stated and supported

(x) Resources properly identified and referenced

(x) Resource documentation appropriate for this application

(x) Input data explicitly stated

(x) Input data verified to be consistent with original source

(x) Geometric model adequate representation of actual geometry

$(x)$ Material properties appropriate and reasonable

(WA Mathematical derivations checked including dimensional consistency

(w) Hand calculations checked for errors

$(x) \quad$ Assumptions explicitly stated and justified

(X) Computer software appropriate for task and used within range of validity

(WA Use of resource outside range of established validity is justified

$(x)$ Software runstreams correct and consistent with results

(x) Software output consistent with input

WA Results consistent with applicable previous experimental or analytical findings

(x) Results and conclusions address all points and are consistent with task requirements and/or established limits or criteria

(x) Conclusions consistent with analytical results and established limits

(x) Uncertainty assessment appropriate and reasonable

( ) Other (define)

III. Comments:

IV. REVIEWER: J. S, DATE: $9-29-98$ 


\section{DISTRIBUTION SHEET}

\begin{tabular}{|c|c|c|c|c|c|}
\hline \multirow{2}{*}{$\begin{array}{l}\text { To } \\
\text { DISTRIBUTION }\end{array}$} & \multirow{2}{*}{\multicolumn{3}{|c|}{$\begin{array}{l}\text { From } \\
\text { Criticality and Shielding }\end{array}$}} & \multicolumn{2}{|l|}{ Page 1 of 1} \\
\hline & & & & \multicolumn{2}{|c|}{ Date $9 / 29 / 98$} \\
\hline \multirow{2}{*}{\multicolumn{4}{|c|}{$\begin{array}{l}\text { Project Title/Work Order } \\
\text { Tank Z-361 Dose Rate Calculations }\end{array}$}} & \multirow{2}{*}{\multicolumn{2}{|c|}{$\begin{array}{ll}\text { EDT No. } & 623014 \\
\text { ECN No. } & \text { NA } \\
\end{array}$}} \\
\hline & & & & & \\
\hline Name & MSIN & $\begin{array}{l}\text { Text } \\
\text { With All } \\
\text { Attach. }\end{array}$ & Text Only & $\begin{array}{l}\text { Attach.f } \\
\text { Appendix } \\
\text { Only }\end{array}$ & $\begin{array}{c}\text { EDT/ECN } \\
\text { Only }\end{array}$ \\
\hline \multicolumn{6}{|l|}{$\begin{array}{l}\text { B \& W Hanford } \\
\text { D. M. Bogen } \\
\text { D. L. Gardner } \\
\text { S. E. Huneycutt } \\
\text { R. S. Jansons }\end{array}$} \\
\hline $\begin{array}{l}\text { Fluor Daniel Northwest } \\
\text { J. P. Estrellado, Jr. } \\
\text { D. P. Hughes } \\
\text { J. S. Lan } \\
\text { R. F. Richard } \\
\text { G. L. Rippy }\end{array}$ & $\begin{array}{l}\text { B4-44 } \\
\text { T5-57 } \\
\text { B4-44 } \\
\text { B4-44 } \\
\text { T5-47 }\end{array}$ & $\begin{array}{l}x \\
x \\
x \\
x \\
x\end{array}$ & & & \\
\hline Central Files (Orig. +2 ) & B1-07 & $x$ & & & \\
\hline
\end{tabular}

